\title{
Theoretically Investigation On The Roughness Profile of The Machined Surface After Flank Milling
}

Jixiong Fei ( $\square$ tjujackfei@163.com )

Institute of Machinery Manufacturing Technology

Bin Lin

Tianjin University

Shuai Yan

Tianjin University

\section{Research Article}

Keywords: Milling, roughness profile, surface topography, modelling

Posted Date: October 22nd, 2021

DOI: https://doi.org/10.21203/rs.3.rs-979726/v1

License: (1) This work is licensed under a Creative Commons Attribution 4.0 International License.

Read Full License 


\title{
Theoretically investigation on the roughness profile of the machined
}

\section{surface after flank milling}

\author{
Jixiong $\mathrm{Fei}^{{ }^{*}}$, Bin $\operatorname{Lin}^{2 *}$, Shuai Yan ${ }^{2}$ \\ ${ }^{1}$ Institute of Machinery Manufacturing Technology, China Academy of Engineering Physics, Chengdu, \\ Sichuan, P. R. China. \\ ${ }^{2}$ Key Laboratory of advanced and machining Technology of Ministry of Education, Tianjin University, \\ Tianjin 300072, P. R. China \\ Corresponding author: Jixiong Fei and Bin Lin \\ Phone: (+86)-022-27404915 \\ Fax: (+86)-022-27404915 \\ E-mail (Jixiong Fei): tjujackfei@163.com \\ E-mail (Bin Lin): linbin@tju.edu.cn \\ E-mail (Shuai Yan):yanshuai163@163.com
}

\begin{abstract}
In this study, the roughness profile of a machined surface obtained via a flank milling process is thoroughly investigated through theoretical modeling and experimental demonstrations. First, the roughness profile of a machined surface generated by a single-tooth end milling cutter along a straight path is considered (without helical angle). The trajectory of a point on the cutting edge is constructed according to the cutting kinematics, and the roughness profile of the flank surface is theoretically extracted from the trajectory. The surface topography is constructed by integrating the roughness profile along the axial direction of the cutter. Based on the constructed roughness profile model, the effects of cutting parameters on the roughness profile are discussed, including those of the cutting speed, radial depth of the cut, and feed rate. In addition, the effects of cutter geometries including the cutter tooth number, tooth spacing angle, and helical angle on the roughness profile and surface topography are discussed. Further, roughness profiles are constructed for cutter feeds along different tool paths, such as round and curved paths. Finally, experiments are conducted to verify the method developed in this study. The results show that the roughness profile obtained from testing matches well with the theoretically modeled profile.
\end{abstract}


Moreover, the methodology for constructing the roughness profile is compared to an existing approach, which shows that the method in this study is significantly faster.

\section{Keywords:}

Milling, roughness profile, surface topography, modelling,

\section{Introduction}

Surface topography or texture of a workpiece is related to its surface quality. It will affect the tribology as well as wear properties of the workpiece. Smooth surface of a workpiece is always required in many engineering practices, especially in the precision engineering. Thus, it is necessary to investigate the topography or texture of a workpiece after being manufactured.

In recent years, the surface topography of a workpiece obtained via milling process, a widely used material removal technique in the aerospace, mold industry as well as other metal cutting industries, has gained more and more attention. Many investigations were dedicated to develop approaches to construct the machined surface topography after milling process and to analyze the factors that affect the surface topography. As to the methods of constructing the surface topography, the one that based on milling kinematics is commonly used. In this method, a set of planes that vertically pass through the cutting tool axis were constructed at first. Then, the roughness profile determined by the trajectories of the cutter edge points in each plane was computed. Finally, the surface topography was obtained by fitting all the surface profiles. For this method, the key to construct the surface topography is to compute the roughness profile in each plane.

So far, different methods to compute the roughness profile were proposed. For example, Arizmendi et al. [1] proposed a discretization method to construct the roughness profile. In their method, a set of lines were equally distributed along the milling path, and the lines were perpendicular to the cutting path. The lines intersected the trajectories and resulted in a set of intersection points. Then, intersection points that belong to the roughness profile were extracted and used to fit the profile. The schematic of the method is show in the Fig. 1. The roughness profile is consisted of a bunch of small arcs that belong to trochiodal trajectories between two adjacent intersection points. Similar to the method developed by Arizmendi et al. [1], Arizmendi et al. [2], Quinsat et al. [3], Layegh and Lazoglu[4] modeled the machined surface topography after 
ball end milling process. Arizmendi and Jiménez [5] modeled the surface topography generated after face milling process. Instead of trochoidal arcs, Buj-Corral et al. [6] approximated the surface profile by circular arcs that equally distributed along the milling path. The distance between two adjacent arcs equals to the feed rate.

(a)

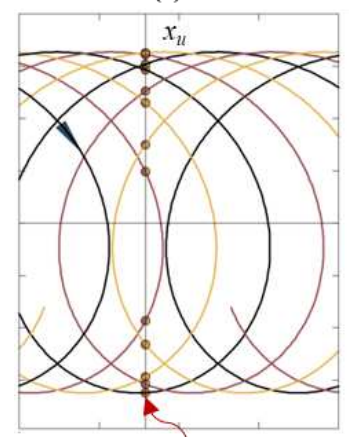

Point belong to roughness profile (b)

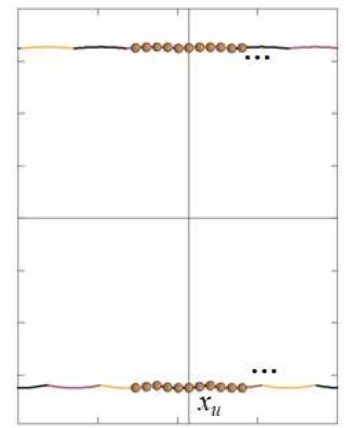

Fig. 1 The schematic to obtain the surface roughness profile [1].

Based on the roughness profile construction methods as reviewed, the effect of cutting parameters such as feed rate $[7,6]$, the effect of tool geometry parameters including helical angle $[7,6]$, the effect of tool error induced by the tool wear [8,9] or tool deflection $[7,9]$, the effect of tool setting error including tool axis offset $[10,6,2]$, tool tilt $[10,4]$ and run out $[7,4,9]$, the effect of tool posture such as tool inclination $[9,4]$, the tool vibration $[1,11]$ on the surface topography were discussed.

In the literatures mentioned above, different methods to construct the roughness profile were developed. Some of the methods are numerical while the rest of them are semi-analytical. Yet none of them tried to understand the roughness profile as well as to analyze the influence of the cutting parameters and the cutter geometries theoretically. Besides, in all the investigation the cutter was assumed to move along a straight path. However, the cutter will move along a curve path when milling a workpiece with complex surface. In addition, the existed methods are time cost to construct the surface topography. For example, it took $5 \mathrm{~h}$ to construct the surface topography within an area about $6 \mathrm{~mm}^{2}[4]$. Henceforth, this investigation focuses on the problems as mentioned and tries to develop an analytical method to construct the roughness profile and to analyze the influence other factors such as cutting parameters and cutter geometry based on the developed method. The rest of the paper is arranged as follows: Section 2 constructs the roughness profile of a very simple case. Based on the simple case, the effect of cutting parameters on the 
roughness profile is discussed in Section 3, the effect of the cutter geometry on the roughness profile is discussed in Section 4 while the roughness profile when the cutter moves along curve is discussed in Section 5. Experiments are conducted in the Section 6 to verify the developed roughness profile model. The whole paper is concluded in Section 7.

\section{Roughness profile generation by the cutter with a single straight tooth}

After flank milling process, two surfaces are generated, namely a bottom and a side face (as shown in Fig. 2 (a)). Only the roughness profile of the side face will be considered in this paper. For simplicity, the milling cutter is assumed to be with a single and straight tooth. The cutter radius is assumed to be $r$. The cutting parameters are assumed to be axial depth of cut $a_{p}$, the radial depth of cut $a_{e}$, the feed velocity $v_{f}$, the spindle speed $n$, the angular rotational speed $\omega$ respectively. Down milling is employed during modelling. During milling process, the tool edge interacts with the workpiece. The surface is generated after the cutting edge sweeps the workpiece materials. Thus, the roughness profile of the machined surface is related to the trajectory of the cutting edge points.
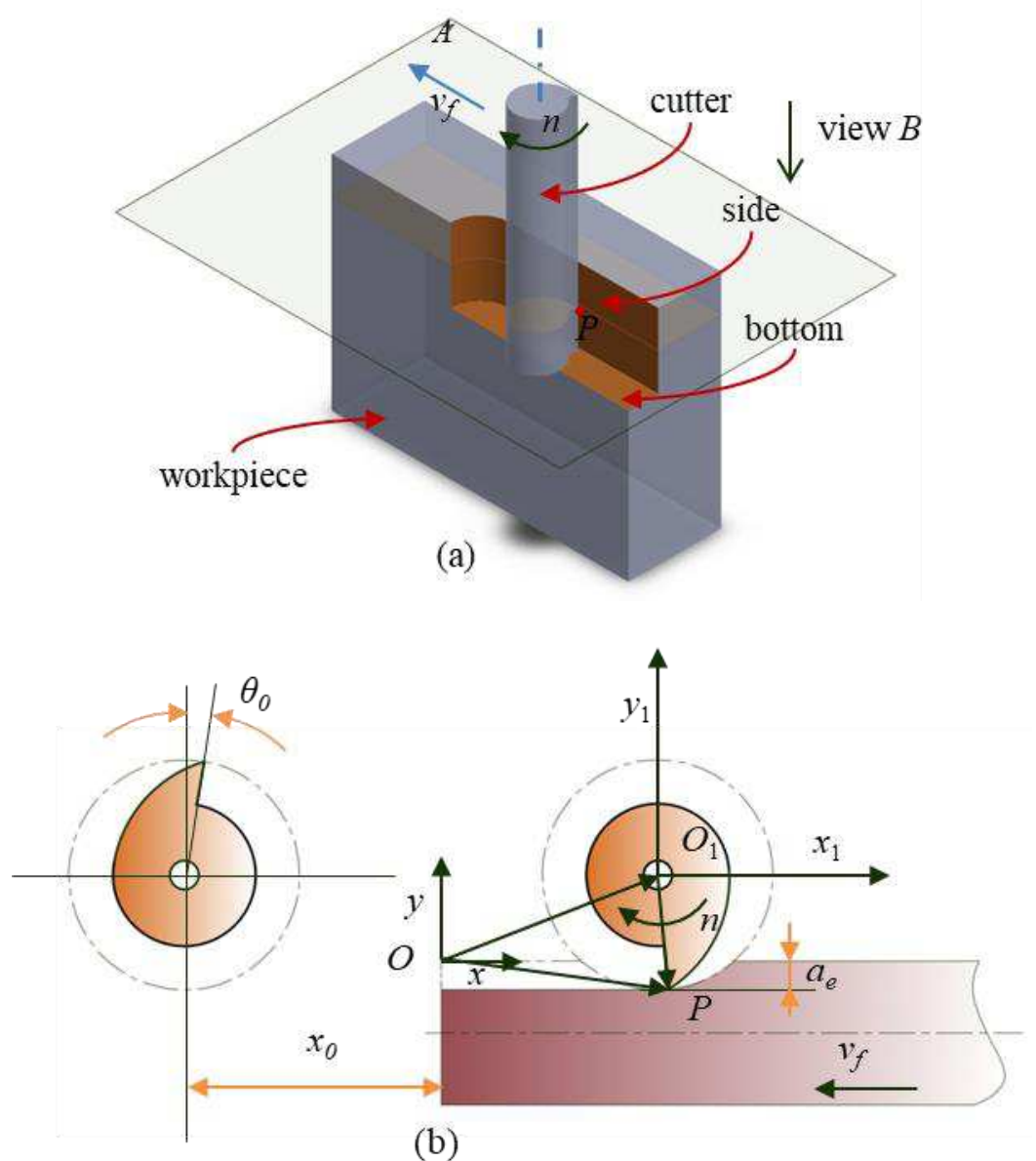
Fig. 2 The schematic diagram of cutter edge point motion: (a) 3D view of the side milling; (b) view from B.

\subsection{The trajectory of a point on the cutting edge}

As mentioned before, the roughness profile is related to the trajectory of the cutting edge point. Thus, the trajectory equation of a point on the cutting edge is derived. Let $P$ denote a point on the cutting edge as shown in the Fig. 2 (a). Let a plane $A$ vertically pass point $P$. The trajectory of the point $P$ locates in this plane $A$. To derive its trajectory, a coordinate system $x-O-y$ is constructed as shown in Fig. 2 (b). Another coordinate system $x_{1}-O_{1}-y_{1}$ is placed at the cutter center with its $x$ axis align with the feed direction, as shown in the Fig. 2 (b). The displacement vector of point $P$ is obtained according to following relationship

$$
\overline{O P}=\overline{O O_{1}}+\overline{O_{1} P}
$$

where

$$
\left\{\begin{array}{l}
\overline{O O_{1}}=\left(v_{f} t+x_{0}, r-a_{e}\right) \\
\overline{O_{1} P}=(r \sin \theta, r \cos \theta)=\left(r \sin \left(\omega t+\theta_{0}\right), r \cos \left(\omega t+\theta_{0}\right)\right)
\end{array}\right.
$$

in which $\theta_{0}$ means the angle between the point $P$ and the $y_{1}$ direction at the initial moment as shown in Fig. 2. $x_{0}$ means the distance between the cutter center and the workpiece at the initial moment.

Substituting expressions of $\overline{O O_{1}}$ and $\overline{O_{1} P}$ into Eq.(1), the trajectory equation of the point $P$ is obtained as

$$
\left\{\begin{array}{l}
x_{P}=v_{f} t+x_{0}+r \sin \left(\omega t+\theta_{0}\right) \\
y_{P}=r-a_{e}+r \cos \left(\omega t+\theta_{0}\right)
\end{array}\right.
$$

Fig. 3 shows the trajectory of the single tooth cutter when the initial start angle is $0^{\circ}$. From the figure, it can be seen that the roughness profile is consisted of the trochoidal arcs among the lowest self-intersection points of the trajectory (only when the self-intersection points locate under the un-machined surface). Henceforth, it is necessary to solve the coordinates of the lowest self-intersection points of the trajectory for construction of the roughness profile. 


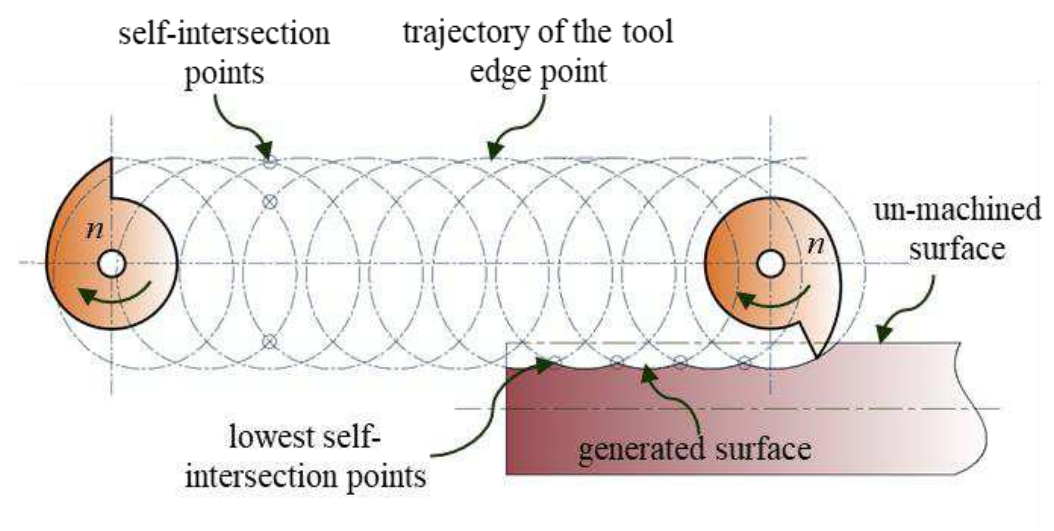

Fig. 3 Trajectory of the point $P\left(r=4 \mathrm{~mm}, a_{e}=1 \mathrm{~mm}, n=1000 \mathrm{rpm}, v_{f} / v_{c}=0.1, x_{0}=-15 \mathrm{~mm}\right)$.

\subsection{Solve the lowest self-intersection points of the trajectory}

Assuming that the coordinate of a self-intersection point is denoted by $\left(x_{1}, y_{1}\right)$, the initial time instant that this self-intersection occurs is $t_{1}\left(t_{1}>0\right)$. After a time period $\Delta t(\Delta t>0)$, the trajectory will come back to itself $\left(x_{1}, y_{1}\right)$ because of self-intersection. Substituting the self-intersection point coordinate $\left(x_{1}, y_{1}\right)$, the time instant $t_{1}$ and the time period $\Delta t$ into Eq.(2) results in

$$
\left\{\begin{array}{l}
r \sin \left(\omega t_{1}+\theta_{0}\right)=v_{f} \Delta t+r \sin \left(\omega\left(t_{1}+\Delta t\right)+\theta_{0}\right) \\
r \cos \left(\omega t_{1}+\theta_{0}\right)=r \cos \left(\omega\left(t_{1}+\Delta t\right)+\theta_{0}\right)
\end{array}\right.
$$

Let $\omega_{1} t+\theta_{0}=\varphi, \omega \Delta t=\Delta \varphi$. Eq.(3) is rewritten as

$$
\left\{\begin{array}{l}
r \sin \varphi=v_{f} \Delta t+r \sin (\varphi+\Delta \varphi) \\
r \cos \varphi=r \cos (\varphi+\Delta \varphi)
\end{array}\right.
$$

From the lower equation of Eq.(4), it can be obtained that

$$
\sin \left(\frac{\Delta \varphi}{2}+\varphi\right) \sin \left(\frac{\Delta \varphi}{2}\right)=0
$$

which results in

$$
\Delta \varphi=2(k \pi-\varphi) \text { or } \Delta \varphi=2 k \pi(k=1,2,3 \ldots)
$$

$\Delta \varphi=2 k \pi$ is an inappropriate solution because it will lead to $v_{f} \Delta t=0$ and thus $\Delta t=0$. This is contradict to the fact that $\Delta t>0$. Therefore, only $\Delta \varphi=2(k \pi-\varphi)$ is the solution of Eq.(5).

From Eq.(4), the following relationship can be obtained

$$
\sin (\varphi+\Delta \varphi)=-\frac{v_{f} \Delta \varphi}{2 v_{c}}
$$

in which $v_{c}=\omega r$. Detail derivation of this equation is given in the appendix. From Eq.(6), $\Delta \varphi$ 
$=2(k \pi-\varphi)$, substitute it into Eq.(7) leads to

$$
\sin \varphi=\frac{v_{f}}{v_{c}}(k \pi-\varphi)
$$

The trajectory of the point $P$ has self-intersection points as long as Eq.(8) has solutions. Clearly, $\varphi=k \pi$ is an solution of the Eq.(8), but it means not that tooth trajectory will intersect itself when the cutter rotates an angle of $k \pi$, because when $k \pi$ is substituted into the first equation of Eq.(6), it will lead to $\Delta \varphi=0$, which is contradict to the fact that $\Delta \varphi>0$. Thus, there are no self-intersection points when the cutter tooth rotates $k \pi$.

From the Eq.(8), it can be seen that its left side means a sinusoidal curve while its right side means a bunch of lines. The intersection points between the lines and the sinusoidal curve are the solution of the equation. It can be seen that the intersection between the sinusoidal curve and the lines is related to the slope of the line, i.e. $v_{f} / v_{c}$. when $v_{f} / v_{c} \geq 1$, namely when the feed speed is larger than or equal to the cutting speed, there will be no intersection between the sinusoidal curve and the lines except for the $\varphi=k \pi$, which means that there is no meaningful solutions for Eq.(8) and thus there is no self-intersection points for the tooth trajectory (Eq.(2)). Fig. 4 (a) shows the intersections between the lines and the sinusoidal curve and the self-intersection of the tooth trajectory when $v_{f} / v_{c}=1.5$, namely when the feed speed is larger than the cutting speed. When $v_{f} / v_{c}<1$, namely when the feed speed is smaller than the cutting speed, there will be other intersection points between the sinusoidal curve and the lines except for the $\varphi=k \pi$, which means that there are meaningful solutions for Eq.(8) and thus there are self-intersection points for the tooth trajectory (Eq.(2)). Fig. 4 (b) shows the intersections between the sinusoidal curve and the lines and the self-intersection of the tooth trajectory when the $v_{f} / v_{c}=0.1$. 


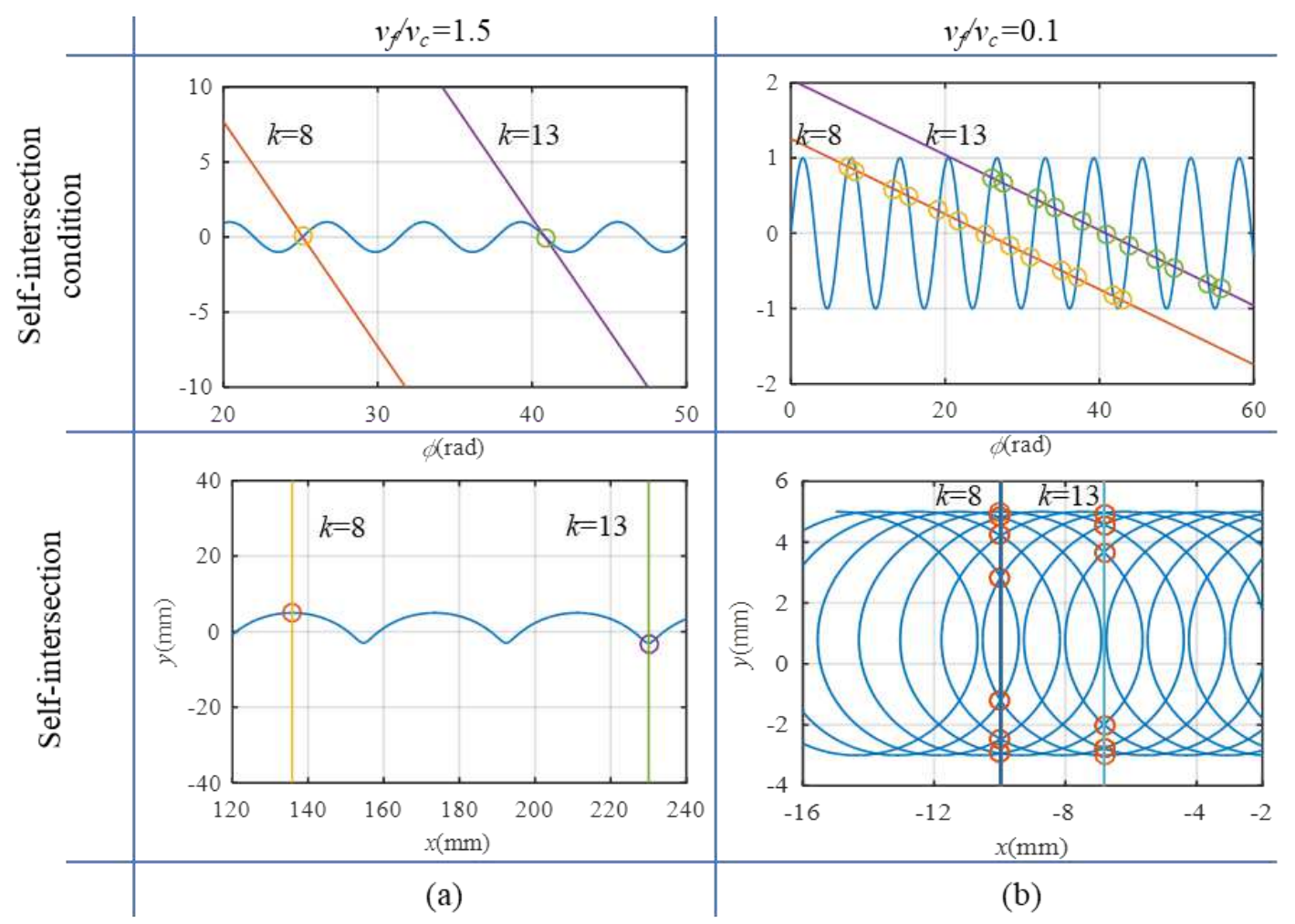

Fig. 4 The self-intersection points: (a) when the $v_{f} / v \geq 1$; (a) when the $v_{f} / v<1$.

In the real milling process, it is unlikely to use a feeding speed $v_{f}$ that larger than or equal to the cutting speed $v_{c}$ since the machined surface is very rough, as shown in the Fig. 5. Besides, it may break the cutting tool. Therefore, small feed speed $\left(v_{f}<v_{c}\right)$ is usually used in real milling process. This investigation will also focus on the case when $v_{f}<v_{c}$. Under this condition, the machined surface generation is related to the lowest self-intersection point as shown in Fig. 3 and Fig. 6. Henceforth, it is necessary to obtain the coordinates of the lowest self-intersection points and the corresponding the time instant that the lowest self-intersection occurs.

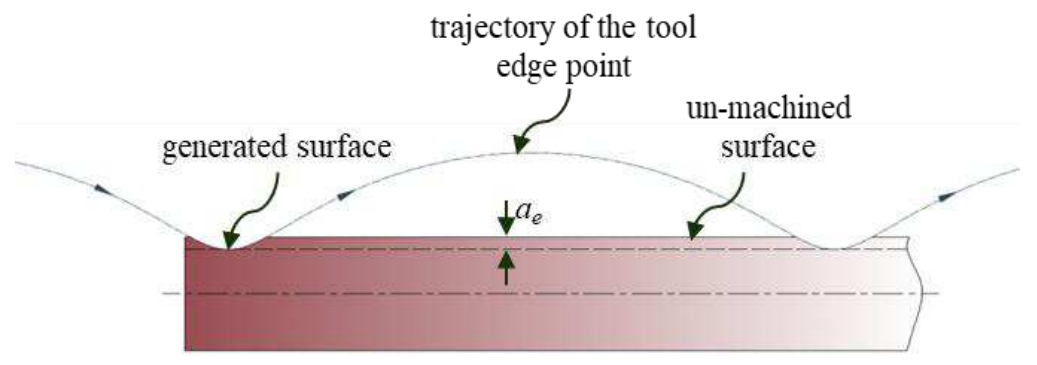

Fig. 5 Machined surface contour when $v_{f} / v=1.5\left(r=4 \mathrm{~mm}, a_{e}=1 \mathrm{~mm}, n=1000 \mathrm{rpm}\right)$. 


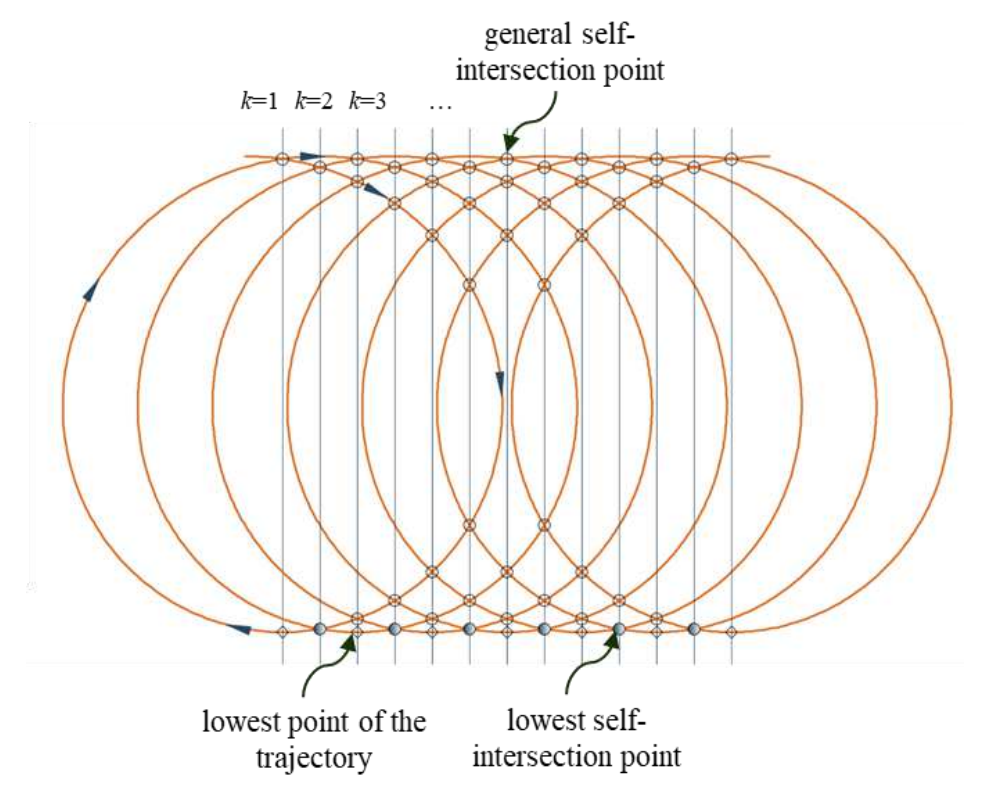

Fig. 6 The lowest self-intersection point ( $\left.v_{f} / v=0.0125, r=4 \mathrm{~mm}, a_{e}=1 \mathrm{~mm}, n=1000 \mathrm{rpm}\right)$.

Define a function as:

$$
f(\varphi)=\sin (\varphi)-\frac{v_{f}}{v_{c}}(k \pi-\varphi) \quad(k=1,2,3 \ldots)
$$

The solutions of Eq. (8) can be thought as the zero points the above function. From Fig. 6, it can be seen that lowest self-intersection points of the trajectory is close to the lowest points of the trajectory. The lowest points occurs when $\varphi=\left(2 k_{1}-1\right) \pi$ where $k_{1}$ is a positive integer. Thus, the values of $\varphi$ at which the line intersect with the sinusoidal curves, also corresponding to the lowest self-intersection points of the trajectory occur, can be approximated by the first order Newton method, in which the initial estimation of the $\varphi_{0}$ can be chosen as $\varphi_{0}=\left(2 k_{1}-1\right) \pi$. Namely

$$
\varphi_{1}=\varphi_{0}-\frac{f\left(\varphi_{0}\right)}{f^{\prime}\left(\varphi_{0}\right)}, \varphi_{0}=\left(2 k_{1}-1\right) \pi
$$

Then the rotation angle at which the lowest self-intersection point occurs is

$$
\varphi_{1}=\left[\left(2 k_{1}-1\right)+\frac{v_{f}}{v_{f}-v_{c}}\right] \pi
$$

Thus, the coordinates of the lowest self-intersection points of the trajectory are obtained by taking $\varphi_{1}$ into Eq.(2), which are written as 


$$
\left\{\begin{array}{l}
x_{1}=v_{f} \frac{\varphi_{1}-\theta_{0}}{\omega}+x_{0}+r \sin \varphi_{1}=v_{f} \frac{\varphi_{1}-\theta_{0}}{\omega}+x_{0}-r \sin \left(\frac{\pi v_{f}}{v_{f}-v_{c}}\right) \\
y_{1}=r-a_{e}+r \cos \varphi_{1}=r-a_{e}-r \cos \left(\frac{\pi v_{f}}{v_{f}-v_{c}}\right)
\end{array}\right.
$$

\subsection{Construct the roughness profile}

The cutter rotation angle range $\varphi_{e}$ between two adjacent self-intersection points is $2 \times\left(2 k_{1}-1\right) \pi-\varphi_{1} \leq$ $\varphi_{e} \leq \varphi_{1}$. The roughness profile of the cutter edge point $P$ can be obtained by taking $\varphi_{e}$ into its trajectory equation. This is true when all the lowest self-intersection points are located under the un-machined part surface as shown in Fig. 3, namely $y_{1}<-a_{e}\left(a_{e}>r\left[1+\cos \left(\varphi_{1}\right)\right] / 2\right)$. However, when the lowest self-intersection points of the trajectory are located above the un-machined workpiece surface as shown in Fig. 7, namely $y_{1}>-a_{e}\left(a_{e}<r\left[1+\cos \left(\varphi_{1}\right)\right] / 2\right)$, there will be some area remain uncut. In this condition, the profile is related to the intersection points between the trajectory and the un-machined workpiece surface. These points can be obtained by letting the $y$ coordinate of the trajectory equal to $-a_{e}$, namely $r-a_{e}+r \cos \left(\omega t+\theta_{0}\right)=r-a_{e}+r \cos \varphi=0$, which results in $\varphi=\arccos \left(-1+a_{e} / r\right)+2 k \pi(k=1,2,3 \ldots)$. The roughness profile is consisted of the trochoidal arcs parts between the two adjacent points intersected by the workpiece surface and the trajectory plus the line that uncut, as shown in Fig. 7 (b).

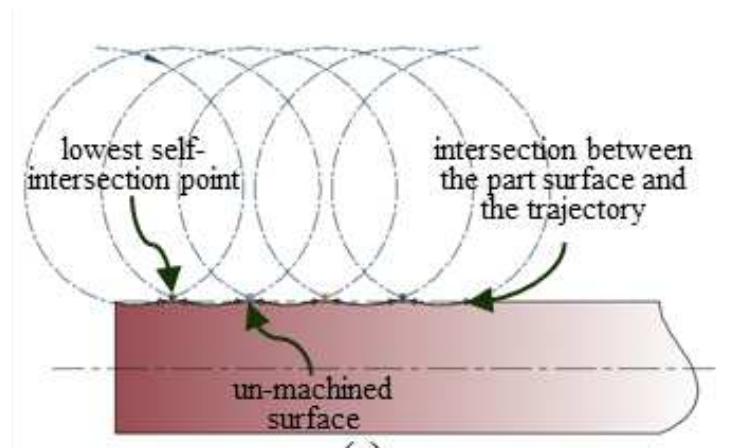

(a)

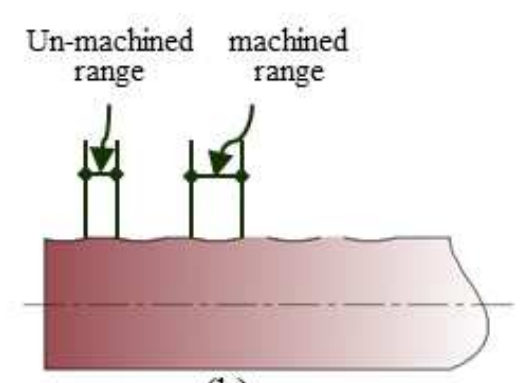

(b)

Fig. 7 Roughness profile when the height of the lowest self-intersection point is above the un-machined workpiece surface: (a) trajectory of the cutter edge point; (b) roughness profile $\left(v_{f} / v_{c}=0.1, r=4 \mathrm{~mm}, a_{e}=1 \mathrm{~mm}, n=1000 \mathrm{rpm}\right)$.

\subsection{Construct the surface topography}

The surface topography is obtained by integrating the roughness profile along the axial direction of the cutter. The axial depth of cut $a_{p}$ is divided into many small pieces as shown Fig. 8 (a). The surface generated by each small cutter edge that corresponding to the small piece of small axial 
depth of cut can be approximated by the roughness profile on the any point of the small cutter edge. A bunch of roughness profiles along the axial direction can be obtained as shown in Fig. 8 (b). The whole surface topography is obtained by fitting all roughness profiles. Fig. 8 (c) shows surface topography when $r=4 \mathrm{~mm}, n=1000 \mathrm{rpm}, v_{f}=0.05 v_{c}$.

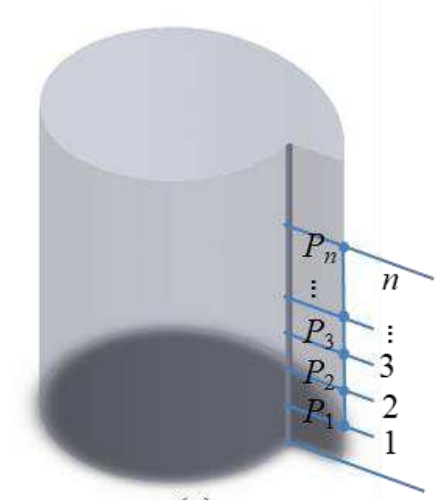

(a)

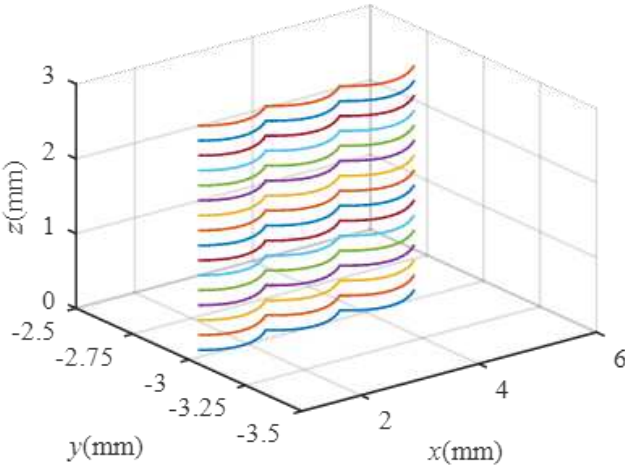

(b)

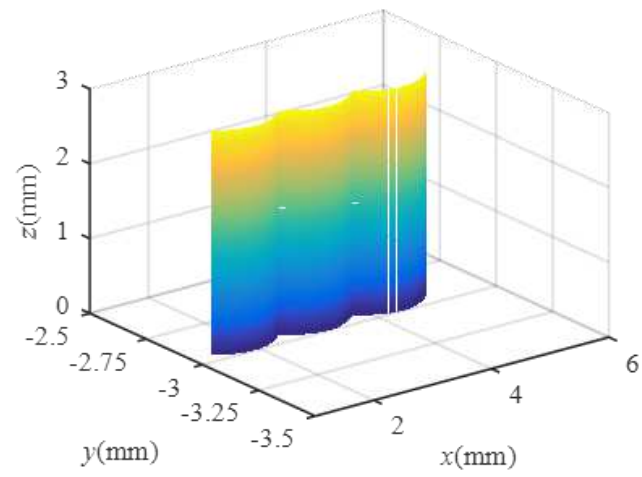

(c)

Fig. 8 Surface topography construction: (a) discretization of the cutter edge; (b) roughness profile of each discretized cutter edge; (c) the fitted surface topography $\left(v_{f} / v_{c}=0.03, r=4 \mathrm{~mm}, a_{e}=1 \mathrm{~mm}, n=1000 \mathrm{rpm}\right)$.

\section{The effect of cutting parameters on roughness profile}

The cutting parameters during milling process include axial depth of cut, radial depth of cut, feed speed and spindle speed. For all these cutting parameters, the axial depth of cut has no effects on the roughness profile due to the straight tooth cutter. The spindle speed will decide the cutting speed according to the relationship $v_{c}=n \pi r / 60$. According to the lower equation of Eq.(11), the height of the lowest self-intersection points (also the height of the roughness $h$ as shown in Fig. 9) is decided by the cutting speed and the feed speed simultaneously. The equation can be rewritten as 


$$
y_{n}=h=r-a_{e}-r \cos \left(\frac{\pi v_{f}}{v_{f}-v_{c}}\right)=r-a_{e}-r \cos \left(\frac{\pi}{1-v_{c} / v_{f}}\right)
$$

From this equation, it can be seen that the height of the roughness profile is decided by the ratio between the feed speed and the cutting speed. The height of the roughness profile respect to the speed ratio is shown in Fig. 9. From the figure, it can be seen that the height increases with the ratio. Roughness profiles of different ratio are shown in Fig. 10. The figure shows that the surface profile is consisted of a series of fundamental element. The fundamental element looks like a part of the parabola. The width of the opening of the element is denoted by $w$ while its height is denoted by $h$. When the ratio increases, the roughness profile becomes higher $(h$ in the figure becomes larger) while the width becomes wider ( $w$ in the figure becomes larger). All these mean that the generated surface will become rougher. Thus, small feed speed should be used during precision stage.

As the increasing of the height $h$, it may exceed the un-machined surface. Under this condition, part of the surface will remain un-machined as shown in Fig. 7. The surface will be very rough. Thus, to avoid this situation, the ratio between feed speed and the cutting speed should be chosen so that $y_{n}=h<-a_{e}$ if the radial depth of cut $a_{e}$ is given, as discussed in the end of Section 2.3 .

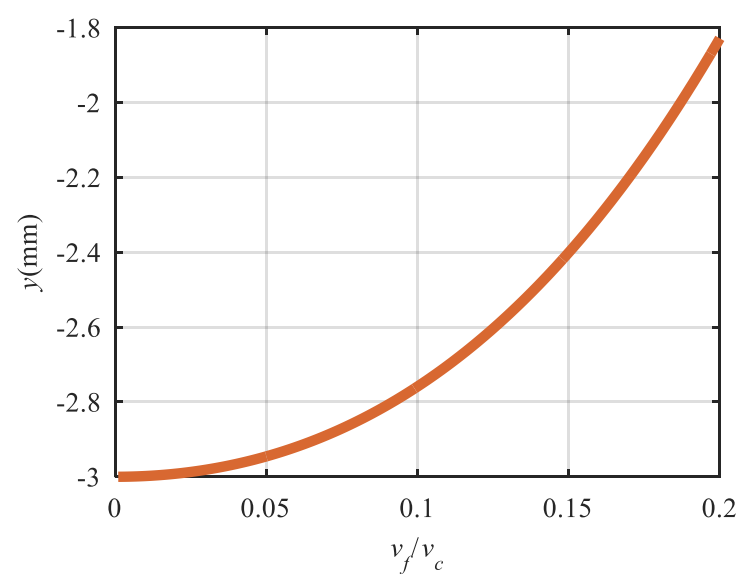

Fig. 9 The height of the roughness profile respect to the ratio between feed speed and cutting speed ( $r=4 \mathrm{~mm}, a_{e}=1$ $\mathrm{mm}, n=1000 \mathrm{rpm})$. 


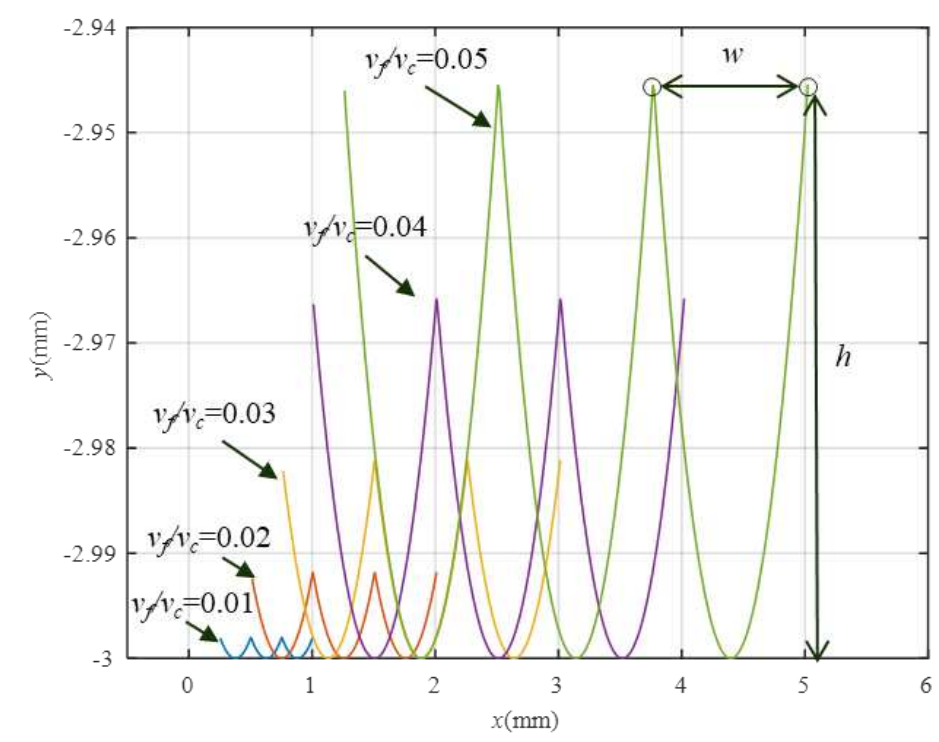

Fig. 10 The roughness profile of different speed ratio ( $\left.r=4 \mathrm{~mm}, a_{e}=1 \mathrm{~mm}, n=1000 \mathrm{rpm}\right)$.

\section{The effect of cutter geometry parameters on roughness profile}

The geometry parameters of an end milling cutter include cutter radius $(r)$, tooth number $(z)$, tooth spacing angle $(\beta)$ and helical angle $(\eta)$. The roughness profile is related to trajectories of the cutting edge points which are affected by the cutter radius, the tooth number, tooth spacing angle and helical angle. The effect of radius on the roughness profile will be reflected by the cutting speed together with the spindle speed as discussed in the Section 3. Henceforth, the effect of tooth number, tooth spacing angle and helical angle will be discussed in detail.

\subsection{The effect of tooth number}

\subsubsection{The trajectories of points on different cutting edge}

In practical milling process, the milling cutter usually has two or more teeth. Thus, it is practical to investigate the machined surface topography generated by the milling cutter with more than one tooth. Assuming that all the teeth are averagely distributed around the cutter, which means that the tooth spacing angle (or pitch angle) is $\beta=2 \pi / z$ ( $z$ is tooth number). For simplicity, the helical angle is still assumed to be zero. The tooth is numbered from 1 to $z$ in a clockwise way. Similar to the procedure in the Section 2.1, let a plane $A$ vertically cross the cutter axis at some height. The plane will intersect with the $z$ cutter edges and thus results in $z$ points, which is marked as $P_{1}, P_{2}, \ldots, P_{z}$ in a clockwise way, as shown Fig. 11 (a). The trajectories for all teeth have the same form but different start position due to the lag angle caused by the tooth spacing angle. In the coordinate 
system as shown in Fig. 11, the trajectory equations for all the cutter edge points is expressed as following

$$
\left\{\begin{array}{l}
x_{P_{i}}=v_{f} t+x_{0}+r \sin \left[\omega t+\theta_{0}-(i-1) \beta\right] \\
y_{P_{i}}=r-a_{e}+r \cos \left[\omega t+\theta_{0}-(i-1) \beta\right]
\end{array}(i=1,2 \ldots, z)\right.
$$

Fig. 12 shows the trajectories when the cutter has different tooth number according to Eq.(13).

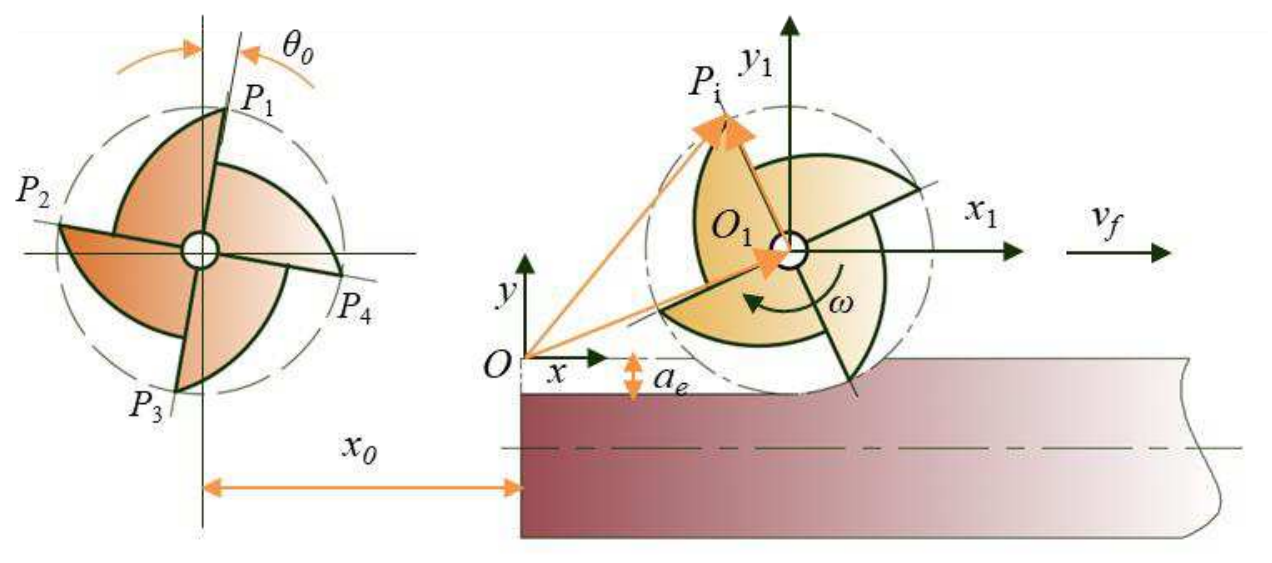

Fig. 11 The cutter with four teeth.

(a)

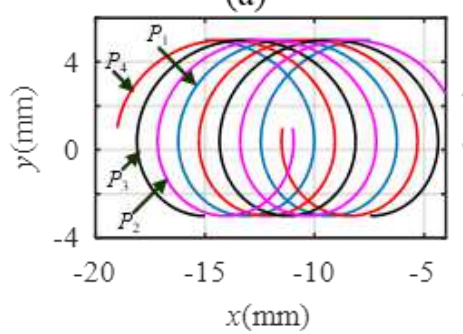

(b)

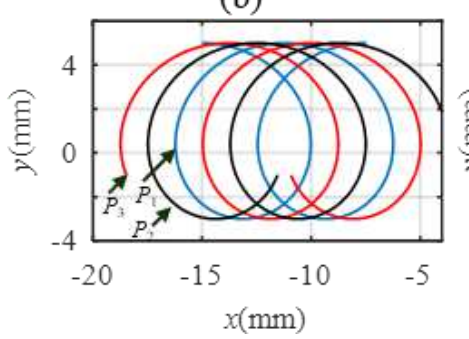

(c)

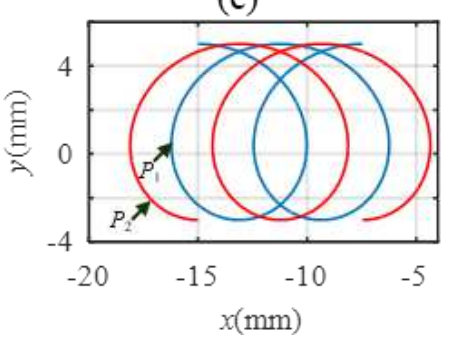

Fig. 12 Trajectory of the cutter with different tooth number ( $r=4 \mathrm{~mm}, n=1000 \mathrm{rpm}, v_{f} / v_{c}=0.05$ ): (a) cutter tooth number $z=4$; (b) cutter tooth number $z=3$; (c) cutter tooth number $z=2$.

\subsubsection{Solve the lowest self-intersection points of the trajectories}

The roughness profile is related to the lowest intersection points of all the cutter teeth trajectories as shown in Fig. 13. The lowest points are generated by intersecting the current tooth trajectory with the succeeded one. Its coordinates can be obtained by solving the intersection points of the corresponding trajectories equations. Assuming that coordinate of intersection point is $\left(x_{i}, y_{i}\right)$, take it into to the corresponding trajectories (Eq.(13)) results in

$$
\left\{\begin{array}{l}
v_{f} t_{1}+x_{0}+r \sin \left[\omega t_{1}+\theta_{0}-(i-1) \beta\right]=v_{f} t_{2}+x_{0}+r \sin \left[\omega t_{2}+\theta_{0}-i \beta\right] \\
r-a_{e}+r \cos \left[\omega t_{1}+\theta_{0}-(i-1) \beta\right]=r-a_{e}+r \cos \left[\omega t_{2}+\theta_{0}-i \beta\right]
\end{array} \quad(i=1,2 \ldots, z)\right.
$$


Solve the lower equation of Eq.(14) results in

$$
\frac{\omega\left(t_{1}+t_{2}\right)+\beta}{2}+i \beta+\theta_{0}=k \pi(k=1,2,3 \ldots) \text { or } \frac{\omega\left(t_{2}-t_{1}\right)-\beta}{2}=k \pi(k=-1,-2,-3 \ldots)
$$

If $\omega\left(t_{1}-t_{2}\right) / 2-\beta / 2=k \pi$, then $t_{1}=(2 k \pi+\beta) / \omega+t_{2}$, take it into upper equation of the Eq.(14) results in

$$
v_{f}\left(t_{1}-t_{2}\right)=0
$$

which means that $t_{1}=t_{2}$, however, $t_{1}=t_{2}$ is impossible. Thus, only when $\omega\left(t_{1}+t_{2}\right) / 2+\beta / 2+i \beta+\theta_{0}=k \pi$, i.e $t_{1}=\left(2 k \pi+(2 i-1) \beta+2-2 \theta_{0}\right) / \omega-t_{2}$, the two trajectories have intersection points. Substitute $t_{1}$ into upper equation of Eq.(14) results in

$$
\frac{v_{f}}{v_{c}}\left(k \pi+\left(i-\frac{1}{2}\right) \beta-\left(\omega t_{2}+\theta_{0}\right)\right)=\sin \left(\omega t_{2}+\theta_{0}-(i-1) \beta\right)
$$

from which one can solve $t_{2}$ and thus the $t_{1}$

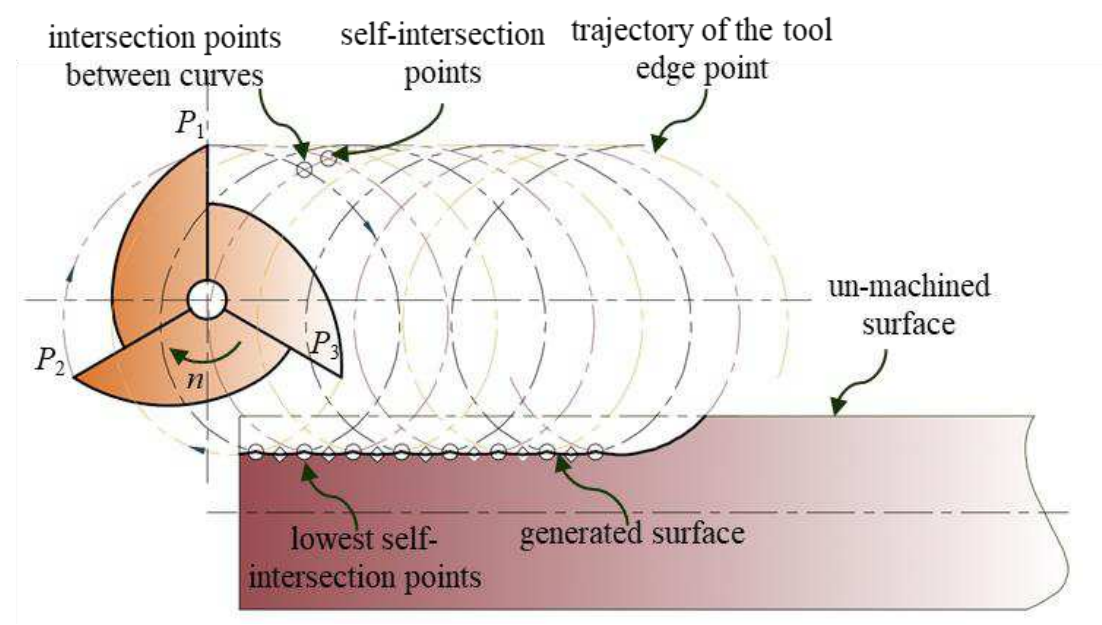

Fig. 13 The lowest intersection points among the trajectories ( $\left.r=4 \mathrm{~mm}, n=1000 \mathrm{rpm}, v_{f} / v_{c}=0.05\right)$.

From Eq.(17), one can solve the angle at which the intersection occurs. The angle values can be thought as the zero points of the following function

$$
f(\varphi)=\sin (\varphi-(i-1) \beta)-\frac{v_{f}}{v_{c}}\left(k \pi+\left(i-\frac{1}{2}\right) \beta-\varphi\right)
$$

in which, $\varphi=\omega t_{2}+\theta_{0}$.

The function $f(\varphi)$ has many zero points. However, only the one corresponding to the lowest intersection points occur is meaningful since only the lowest intersection points as shown in Fig. 13 is related to the roughness profile. Each lowest intersection point is close to the location when the cutter tooth reaches to the lowest position at $y$ direction, at which the rotation angle of the 
cutter equals to $(2 k-1) \pi$, which means that the zero points are close to $(2 k-1) \pi$. The approximation values of the zero points can be obtained according to the Newton formulation. The first iteration is written as

$$
\varphi_{1}=\varphi_{0}-\frac{f\left(\varphi_{0}\right)}{f^{\prime}\left(\varphi_{0}\right)}
$$

Because that $\varphi=(2 k-1) \pi$ is close to the zero points, it can be regarded as initial value of first iteration, namely, $\varphi_{0}=(2 k-1) \pi$. The zero point value of the first iteration is obtained as

$$
\varphi_{1}=(2 k-1) \pi-\frac{\sin [(i-1) \beta]+\frac{v_{f}}{v_{c}}\left(i-\frac{1}{2}\right) \beta}{\cos [(i-1) \beta]+\frac{v_{f}}{v_{c}}}
$$

The coordinates of the lowest intersection points can be obtained by taking the $\varphi_{1}$ into the trajectory equation (Eq.(14)). First iteration may give the values of the zero points without enough accuracy. In such case, one can do several iterations according to the Newton formulation (Eq.(21)) to obtain the zero values with enough accuracy.

$$
\varphi_{n+1}=\varphi_{n}-\frac{f\left(\varphi_{n}\right)}{f^{\prime}\left(\varphi_{n}\right)}
$$

Fig. 14 shows the intersection point with different times of iterations. The first iteration value has larger difference compared to the value with ten times of iteration.

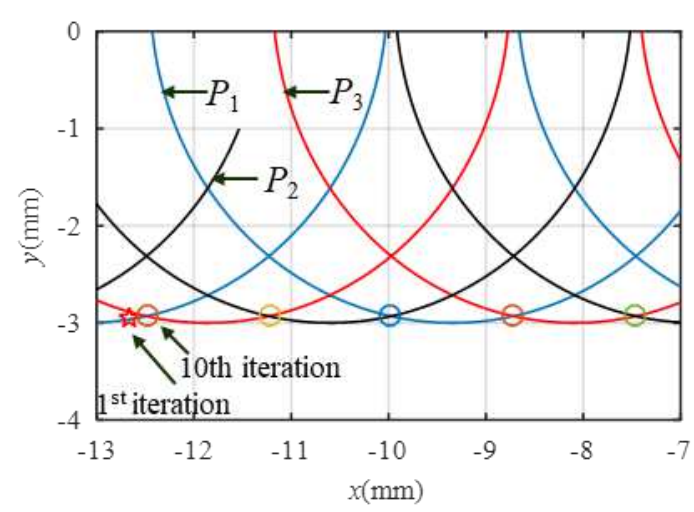

Fig. 14 The approximation of the lowest intersection ( $\left.r=4 \mathrm{~mm}, n=1000 \mathrm{rpm}, v_{f} / v_{c}=0.05\right)$.

After the coordinates of the lowest intersection points are obtained. The rotation angle of the cutter between two adjacent lowest intersection points can also be obtained and thus the roughness profile can be constructed. The surface topography can be constructed by the similar procedures as described in the Section 2.3. 


\subsubsection{The effect of tooth number on roughness profile}

From Eq.(20), it can be seen that the angle at which the lowest intersection points between two adjacent trajectories occurs will be affected by the tooth spacing angle, which is affected by the cutter tooth number. Take it into the $y$ coordinate of the trajectory, the height of the lowest intersection points (also the height of the roughness profile $h$ as shown in Fig. 16) between two adjacent trajectories can be obtained as

$$
y=r-a_{e}-r \cos \left[-\frac{\sin [(i-1) \beta]+\frac{v_{f}}{v_{c}}\left(i-\frac{1}{2}\right) \beta}{\cos [(i-1) \beta]+\frac{v_{f}}{v_{c}}}-(i-1) \beta\right](i=1,2, \ldots, z, k=1,2,3 \ldots)
$$

According to the above equation, the height of the lowest intersection point respect to the tooth number can be analyzed as long as the ratio between the cutting speed and the feed speed is given. Fig. 15 shows the varying trend when the cutter radius $r=4 \mathrm{~mm}$, speed ratio $v_{f} / v_{c}=0.05$, spindle speed $n=1000 \mathrm{rpm}$. The figure shows that the height becomes lower and lower when the cutter teeth number increases, which is similar to the effect of feed speed. Thus, more cutter teeth number will result in smoother surface when the cutting speed is given.

Fig. 16 shows the roughness profile of different tooth number. The profile has the same shape as shown in Fig. 10. The more the teeth number is, the lower the height $(h)$ is and the narrower the opening is $(w)$. Thus, the surface will become smoother.

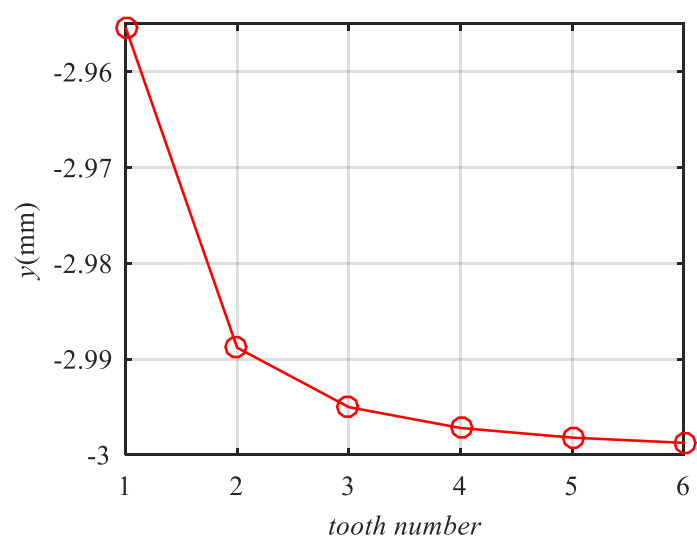

Fig. 15 The height of the intersection point respect to the tooth number ( $\left.r=4 \mathrm{~mm}, n=1000 \mathrm{rpm}, v_{f} / v_{c}=0.05\right)$. 


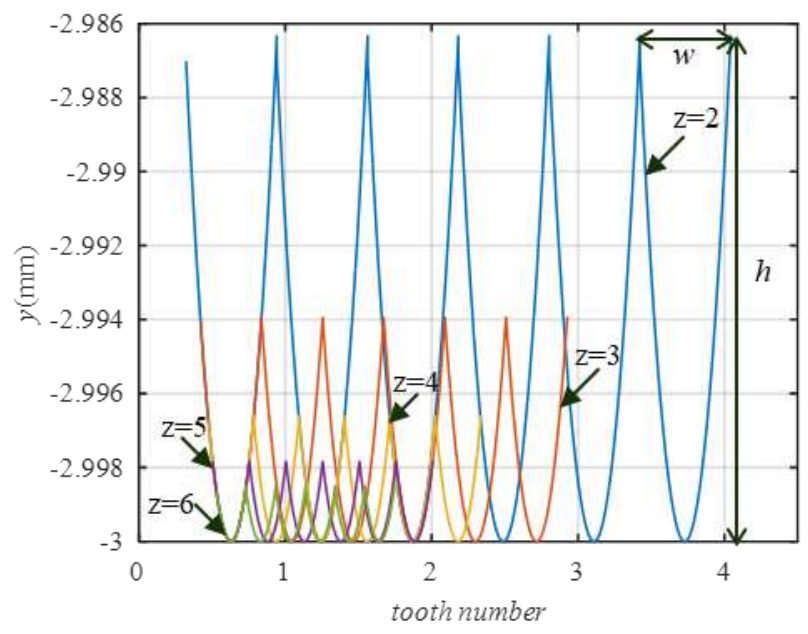

Fig. 16 The surface profile of different cutter tooth number ( $\left.r=4 \mathrm{~mm}, n=1000 \mathrm{rpm}, v_{f} / v_{c}=0.05\right)$.

\subsection{The effect of tooth spacing angle}

Recently, the variable pitch cutter is widely investigated because its effectiveness on chatter suppression [12-15]. Based on the method proposed in the above mentioned sections, this part tries to construct the roughness profile obtained by the variable pitch cutter.

Fig. 17 shows the cutter with constant and variable tooth spacing angle respectively. Fig. 17 (a) is the cutter with constant tooth spacing angle, in which the angle between two adjacent cutter teeth is constant. Fig. 17 (b) is the cutter with variable tooth spacing angle, in which the angle between two adjacent teeth is not equal to each other. For the cutter with variable tooth spacing angle, the tooth spacing angle has the relationship that $\beta_{1}=\beta_{3}$ and $\beta_{2}=\beta_{4}$ for dynamic balance. Fig. 18 shows the trajectories of the cutting edge points and its generated roughness profile for a cutter with variable tooth spacing angle. From the figure, it can be seen that the roughness profile is still related to the lowest intersection points between the trajectories. The height of the lowest intersection points and the distance between two adjacent lowest intersection points are changed due to the variable tooth spacing angle according to Eq.(20).

For simplicity, this part compares the roughness profile of two kinds of four-tooth cutter, one with constant tooth spacing angle while the other one with variable tooth spacing angle, the tooth spacing angle is $77^{\circ}-103^{\circ}-77^{\circ}-103^{\circ}$ and $70^{\circ}-110^{\circ}-70^{\circ}-110^{\circ}$ respectively according to [12]. The roughness profiles of all these three cutters are listed in the Fig. 19. From the figure, the wave crest of the roughness profile for the constant tooth spacing angle has the same height, unlike the one for the variable tooth spacing angle in which the height of the wave crest has different height. 
Besides, the total height of the roughness profile for the variable pitch cutter is larger than the one for the constant pitch cutter. It means that the surface topography generated by the cutter with constant tooth spacing angle is smoother.
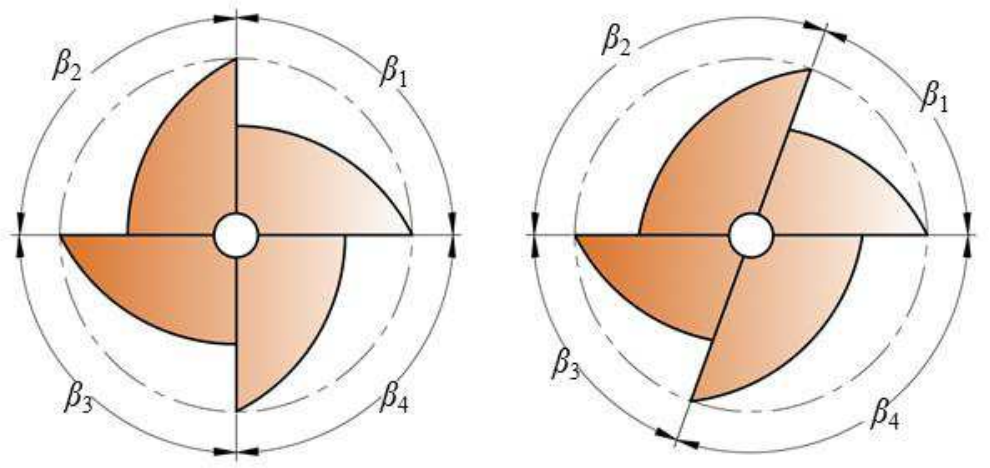

Fig. 17 Cutters with different tooth spacing angles: (a) constant tooth spacing angle; (b) variable tooth spacing angle.

trajectory of the tool edge point

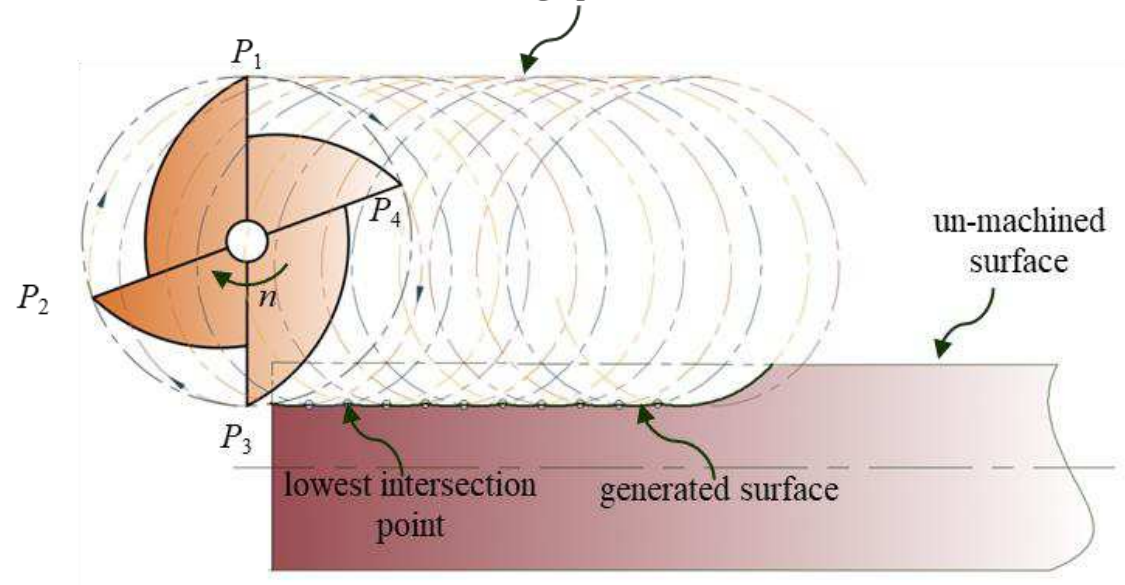

Fig. 18 The tooth trajectories and the lowest intersection points $\left(r=4 \mathrm{~mm}, n=1000 \mathrm{rpm}, v_{f} / v_{c}=0.05\right)$.

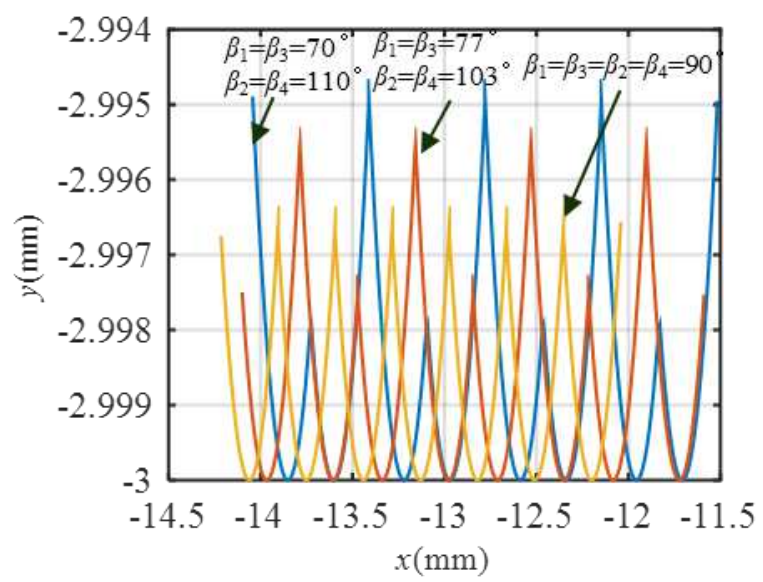

Fig. 19 The roughness profile for the cutter with different tooth spacing angle ( $\left.r=4 \mathrm{~mm}, n=1000 \mathrm{rpm}, v_{f} / v_{c}=0.05\right)$. 


\subsection{The effect of helical angle}

In the above investigation, the cutting edge is assumed to be with zero helical angle. Actually, cutter with helical cutting edge is often used in real milling process. This part will consider the effect the helical angle on the roughness profile and the surface topography. For simplicity, only one cutter tooth is considered. Choose a point $P$ on the cutter edge. The $P$ has a height of $z_{0}$ reference from the bottom cutter center. Let a plane $A$ cross the point $P$ and be vertical to the cutter axis. The cutter edge point at the height of $z_{0}$ has a lag angle compared to the cutting edge point with zero height as shown in Fig. 20. The lag angle is represented by $\theta$, which can be written as

$$
\theta=\frac{z_{0}}{r \tan \eta}
$$

As shown in the Fig. 21, a coordinate system is placed on the center of the cutter at the bottom. $x$ direction of the coordinate system is set to be along the feed direction, $y$ direction of the coordinate system can be obtained by the right-hand rule. Thus, the trajectory of the cutting point $P$ on the cutting edge in plane $A$

$$
\left\{\begin{array}{l}
x_{P_{z_{0}}}=x_{0}+v_{f} t+r \sin \left(\omega t+\theta_{0}+\theta\right) \\
y_{P_{z_{0}}}=y_{0}+r \cos \left(\omega t+\theta_{0}+\theta\right)
\end{array}\right.
$$

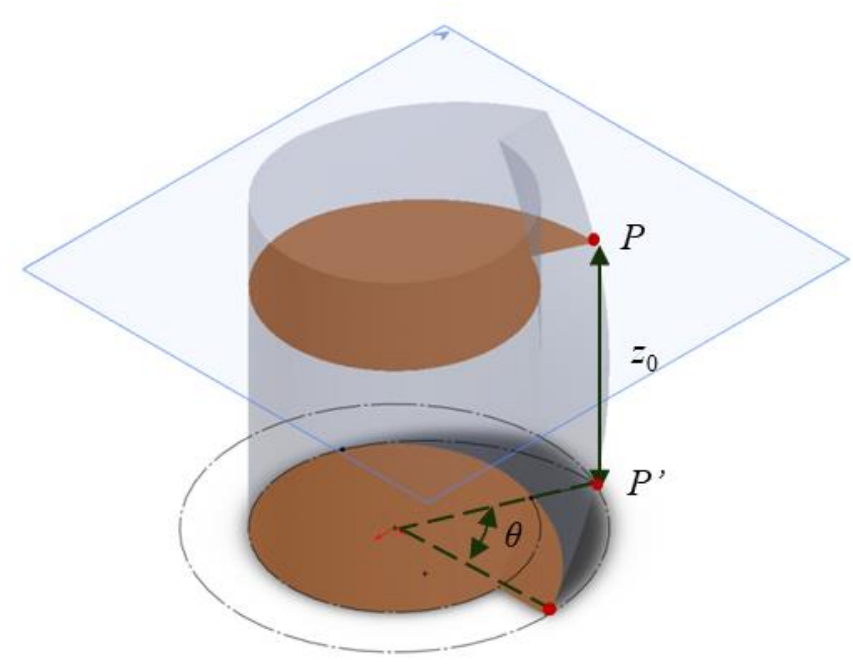

Fig. 20 The lag behand angle $\theta$. 


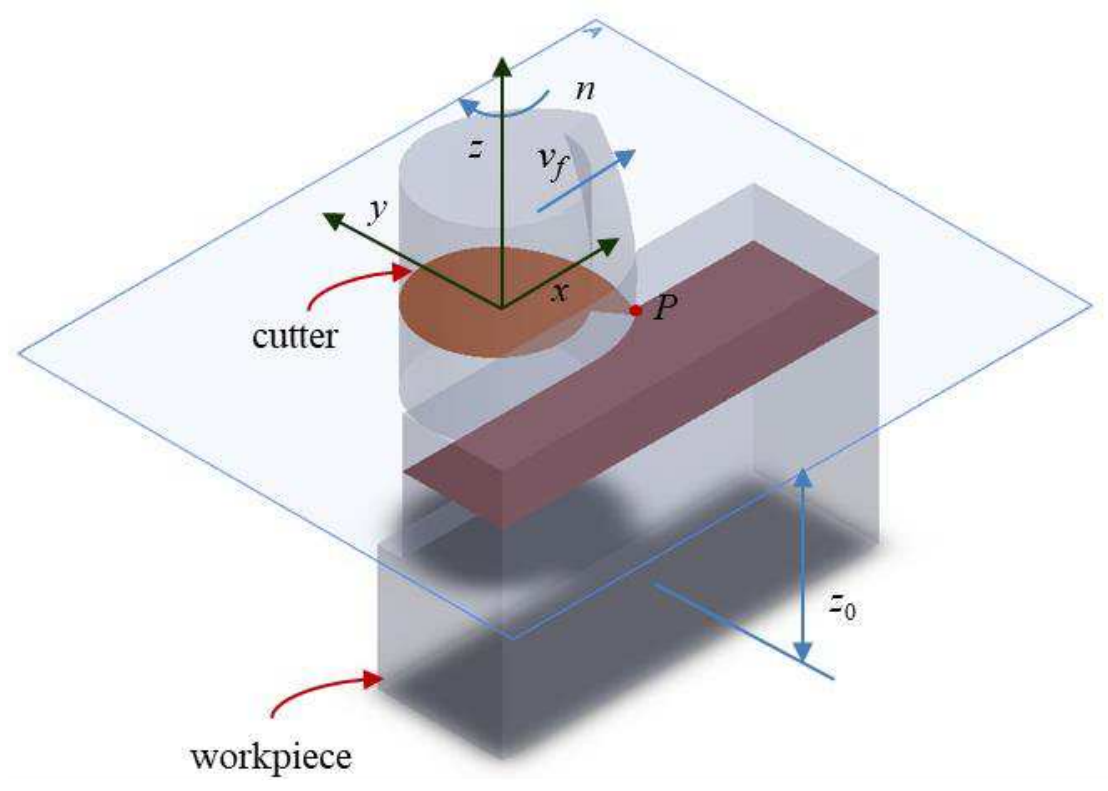

Fig. 21 The coordinate system on the plane $A$.

The roughness profile in plane $A$ can be obtained according to the trajectory of $P$ (Eq.(24)) and the procedures presented in Section 2. As to the whole surface topography, the axial depth of cut is divided into many small parts averagely. At each small part of depth of cut, the cutting edge is assumed to be upright. Thus, the profile for this small part can be obtained according to the procedures presented in Section 2. The whole surface topography can be obtained by integrating all the roughness profiles. As such, it can be seen that the helical angle will affect the phase of trajectory of the cutter edge point, which will affect the position of the lowest intersection points but not the height $(h)$ and the distance $(w)$ between two adjacent intersection points as shown in Fig. 22. There is a shift between the two roughness profiles that generated at two different heights. This shift will be reflected on the surface topography. The peak of the generated surface topography will lie on an oblique line as shown in Fig. 23, which is very different from the surface topography generated with straight cutter tooth as shown in Fig. 8 (c). 


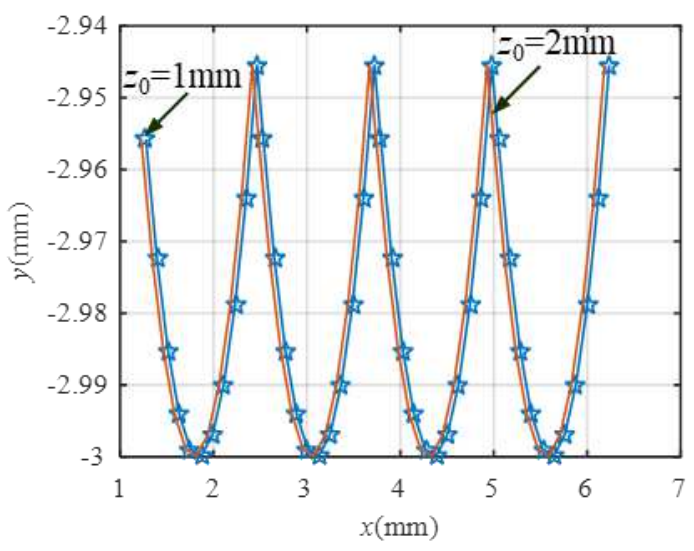

Fig. 22 The surface roughness at different high level ( $\left.r=4 \mathrm{~mm}, n=1000 \mathrm{rpm}, v_{f} / v_{c}=0.05\right)$.

(a)

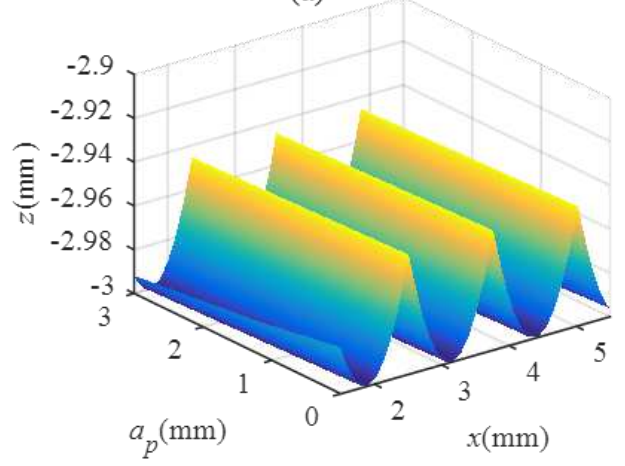

(b)

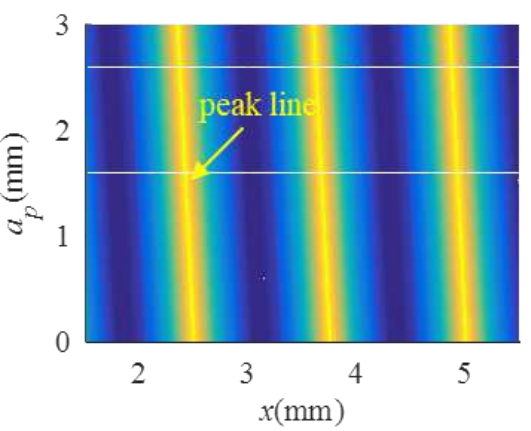

Fig. 23 The surface topography with helical end milling cutter $\left(r=4 \mathrm{~mm}, n=1000 \mathrm{rpm}, v_{f} / v_{c}=0.05, \eta=45^{\circ}\right)$.

\section{The effect of tooth path on roughness profile}

In the above mentioned investigation, the cutter moves along a straight path. However, the cutter will move along curve path during milling the workpiece with complex surface. Therefore, it is significant to construct the roughness profile of the workpiece with curve surface after milling. This part will try to construct the roughness profile that generated by the cutter moves along a curve path and to discuss its effect.

\subsection{Milling along a round path}

Cylindrical workpiece is widely used in the engineering practice. When machining the outer surface of a cylindrical workpiece, the cutter will move along a round path. This part will discuss the roughness profile of the outer surface of a cylindrical workpiece after flank milling process, as shown in Fig. 24 (a). Assume that the radius of the cylindrical workpiece after machining is $R$. For simplicity, the cutter is assumed to be with a single and straight tooth. Two coordinate systems $\left(x-O-y\right.$ and $\left.x_{1}-O_{1}-y_{1}\right)$ as shown in the Fig. 24 (b) are constructed. 


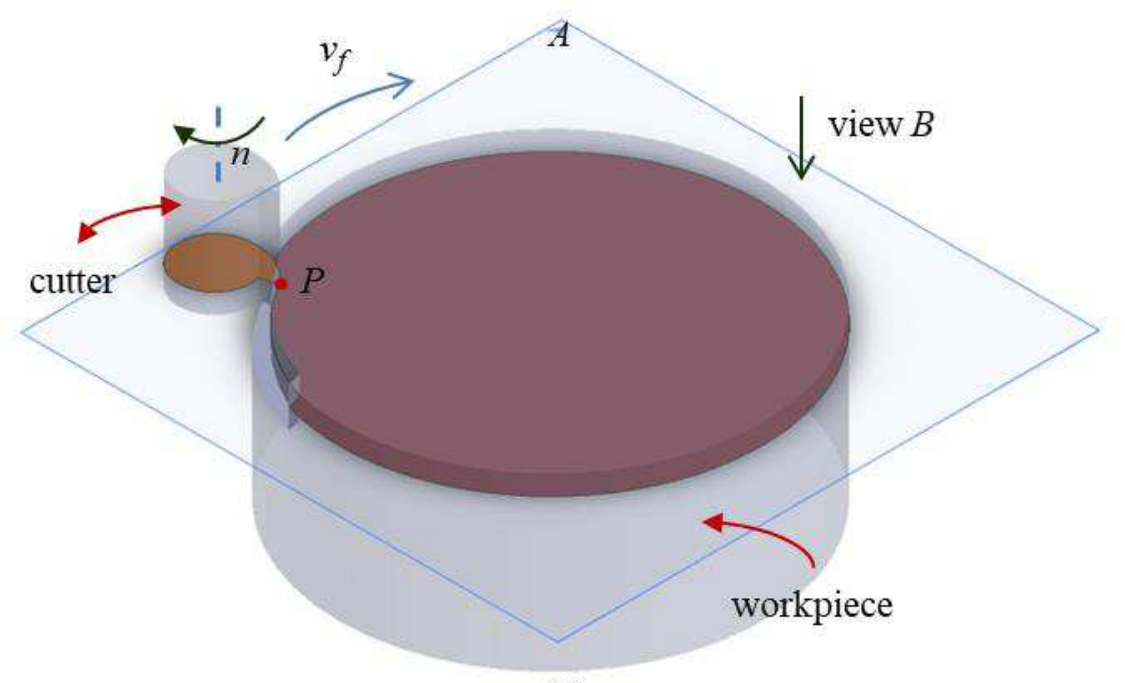

(a)

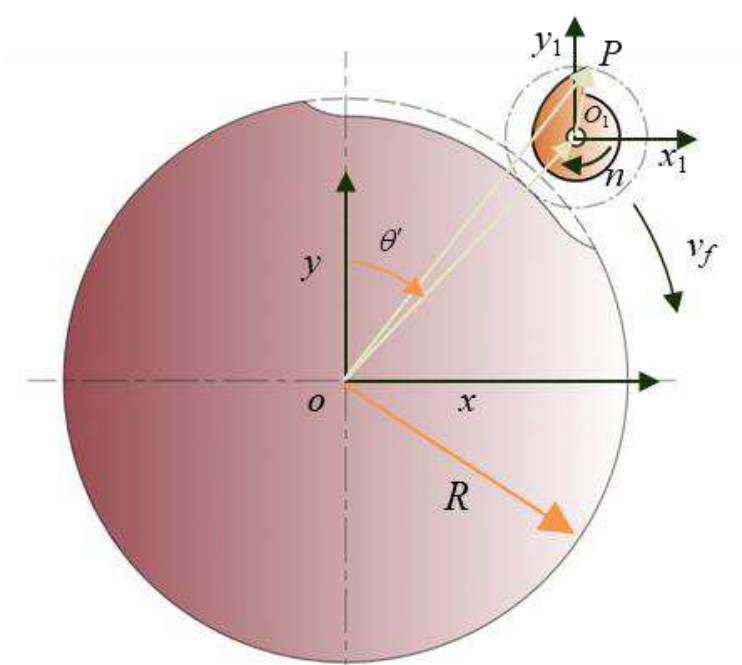

(b)

Fig. 24 Milling cylindrical workpiece with round path: (a) 3D view of the cylinder milling; (b) the coordinate systems to construct the tooth trajectory (view from B).

\subsubsection{The trajectory of a cutting edge point}

To obtain the trajectory of a point $P$ on the cutter edge, following vector relationship must be satisfied during milling process according to Fig. 24 (b).

$$
\overline{O P}=\overline{O O^{\prime}}+\overline{O^{\prime} P}
$$

in which

$$
\left\{\begin{array}{l}
\overline{O O^{\prime}}=\left((R+r) \sin \left(\theta^{\prime}+\theta_{0}^{\prime}\right),(R+r) \sin \left(\theta^{\prime}+\theta_{0}^{\prime}\right)\right) \\
\overline{O^{\prime} P}=\left(r \sin \left(\theta+\theta_{0}\right), r \sin \left(\theta+\theta_{0}\right)\right)
\end{array}\right.
$$

Take the expressions of $\overline{O O_{1}}$ and $\overline{O_{1} P}$ into Eq.(25) results in 


$$
\left\{\begin{array}{l}
x_{p}=(R+r) \sin \left(\theta^{\prime}+\theta_{0}^{\prime}\right)+r \sin \left(\theta+\theta_{0}\right) \\
y_{p}=(R+r) \cos \left(\theta^{\prime}+\theta_{0}^{\prime}\right)+r \cos \left(\theta+\theta_{0}\right)
\end{array}\right.
$$

in which

$$
\theta^{\prime}=\frac{v_{f} t}{R+r}=\omega^{\prime} t, \theta=\omega t
$$

\subsubsection{Solve the self-intersection points and construct roughness profile}

Suppose that the self-intersection occurs at $\left(x_{1}, y_{1}\right)$, the corresponding time instant is $t_{1}$. After time period $\Delta t$, the trajectory will go back to itself. Thus, for the trajectory, it will satisfy

$$
\left\{\begin{array}{l}
x_{p}\left(t_{1}\right)=x_{p}\left(t_{1}+\Delta t\right) \\
x_{p}\left(t_{1}\right)=x_{p}\left(t_{1}+\Delta t\right)
\end{array}\right.
$$

From Eq.(27) and Eq.(26), one can derive that

$$
t_{1}=\frac{k \pi+\theta_{0}-\theta_{0}^{\prime}}{\omega^{\prime}-\omega}-\frac{\Delta t}{2}
$$

Taking Eq.(28) into upper equation of Eq.(27) results in

$$
-\sin \frac{\omega^{\prime} \Delta t}{2}=(-1)^{k} \frac{r}{R+r} \sin \frac{\omega \Delta t}{2},(k=1,2,3 \ldots)
$$

from which one can solve time period $\Delta t$, and thus the time instant $t_{1}$, and thus the coordinates of the self-intersection points of the trajectory. Hence, the self-intersection points of the trajectory is dependent on the solution of Eq.(29).

Both sides of Eq.(29) are sinusoidal curves in the right-half plane. The intersection points of the two sinusoidal curves are infinite. Besides, it is dependent on $k$. Assuming that the solution for Eq.(29) is $\Delta t_{i k 1}$ when $k$ is odd while it is $\Delta t_{i k 2}$ when $k$ is even. All solutions of Eq.(29) can be listed in the following table, in which $0<\Delta t_{1 \mathrm{k} 1}<\Delta t_{2 \mathrm{k} 1}<\ldots<\Delta t_{i k 1}<\ldots, 0<\Delta t_{1 \mathrm{k} 2}<\Delta t_{2 \mathrm{k} 2}<\ldots<\Delta t_{i k 2}<\ldots$

Table 1 the solutions of Eq.(29)

\begin{tabular}{cccccc}
\hline & 1 & 2 & $\ldots$ & $i$ & $\ldots$ \\
\hline$k=1+2 n(n=0,1,2 \ldots)$ & $\Delta t_{1 \mathrm{k} 1}$ & $\Delta t_{2 \mathrm{k} 1}$ & $\ldots$ & $\Delta t_{i k 1}$ & $\ldots$ \\
$k=2 n(n=0,1,2 \ldots)$ & $\Delta t_{1 \mathrm{k} 2}$ & $\Delta t_{2 \mathrm{k} 2}$ & $\ldots$ & $\Delta t_{i k 2}$ & $\ldots$ \\
\hline
\end{tabular}

The roughness profile is related to the self-intersection points of the trajectory as shown in

Fig. 25. Once the related self-intersection points are obtained, the roughness profile can be constructed. Henceforth, the key task is to find the right $\Delta t_{i}$ that corresponding to the self-intersection points of the trajectory in all infinite solutions of Eq.(29), which are shown in the 
above table. Luckily, it can be easily found that the meaningful solutions of Eq.(29) must satisfy

$$
0<\frac{\omega^{\prime} \Delta t}{2}<\pi
$$

which leads to $0<\Delta t<2 \pi / \omega^{\prime}$. Besides,

$$
t_{1}=\frac{k \pi+\theta_{0}-\theta_{0}^{\prime}}{\omega^{\prime}-\omega}-\frac{\Delta t}{2}>0
$$

which means that the $t_{1}$ and $\Delta t$ has a linear relationship.

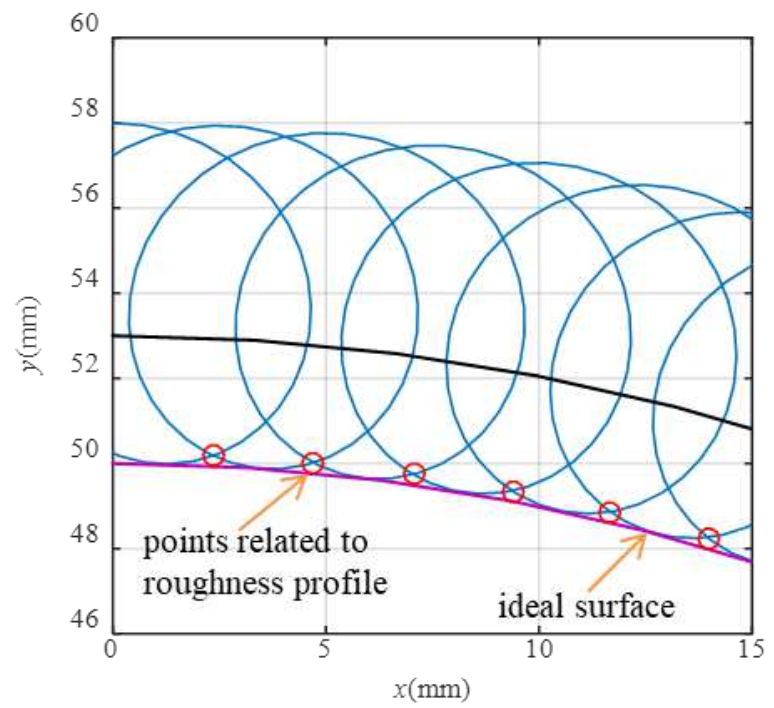

Fig. 25 The self-intersection points of the trajectory $\left(r=4 \mathrm{~mm}, n=1000 \mathrm{rpm}, v_{f} / v_{c}=0.1\right)$.

To make it easier to find the meaningful values of $\Delta t$, a lot of numerical simulation is implemented based on MATLAB. During simulation, the $k$ in the Eq.(29) is chosen to be 1, 2, 3, 4, 5 , while $R=50 \mathrm{~mm}$ and $r=4 \mathrm{~mm}$. The spindle speed is chosen to be $1000 \mathrm{rpm}$. The cutting speed can be decided as $v_{c}=n \pi / 30=418.879 \mathrm{~mm} / \mathrm{min}$. The feed speed is chosen to be $v_{f}=0.1 v_{c}, 0.2 v_{c}, 0.3 v_{c}$ and $v_{c}$ respectively. The ratio between the $\omega^{\prime}$ and $\omega$ can be computed as $r_{\omega}=0.0074,0.0148,0.0222$ and 0.0741 respectively. The simulation results are shown in Fig. 26. The points among the sinusoidal curves (namely the solution of Eq.(29)) are shown in the first column while the corresponding self-intersection points are shown in second column. The magnification of the self-intersection points are shown in third column. And the linear relationship $t_{l}$ and $\Delta t$ are shown in the fourth column of the figure and the circles in the figure mean the intersection moments and corresponding time intervals. From the figure, it can be found that ratio between feed speed and cutting speed $\left(r_{v}\right)$ cannot be too large. There exists a critical value that decides the roughness profile. The critical ratio $r_{v c r i t i c a l}$ lies between 0.2 and 0.3 for this specific simulation case. At this 
critical value, there exists just one intersection point between the two sinusoidal curves, namely $\sin \left(\omega^{\prime} \Delta t / 2\right)$ and $(-1)^{k} r /(R+r) \sin (\omega \Delta t / 2)(k$ is even). When the ratio is larger than this critical value, there will be no self-intersection points on the right hand side of the trajectory. At this condition, there will be part of workpiece surface remain to be uncut (as shown in the third row of Fig. 26), which make the surface is very rough. In the real machining, it is impossible to choose a cutting condition in which the ratio is larger than critical ratio $r_{v c r i t i c a l}$. Henceforth, this paper focus on the condition of $r_{v}<r_{\text {vcritical }}$.
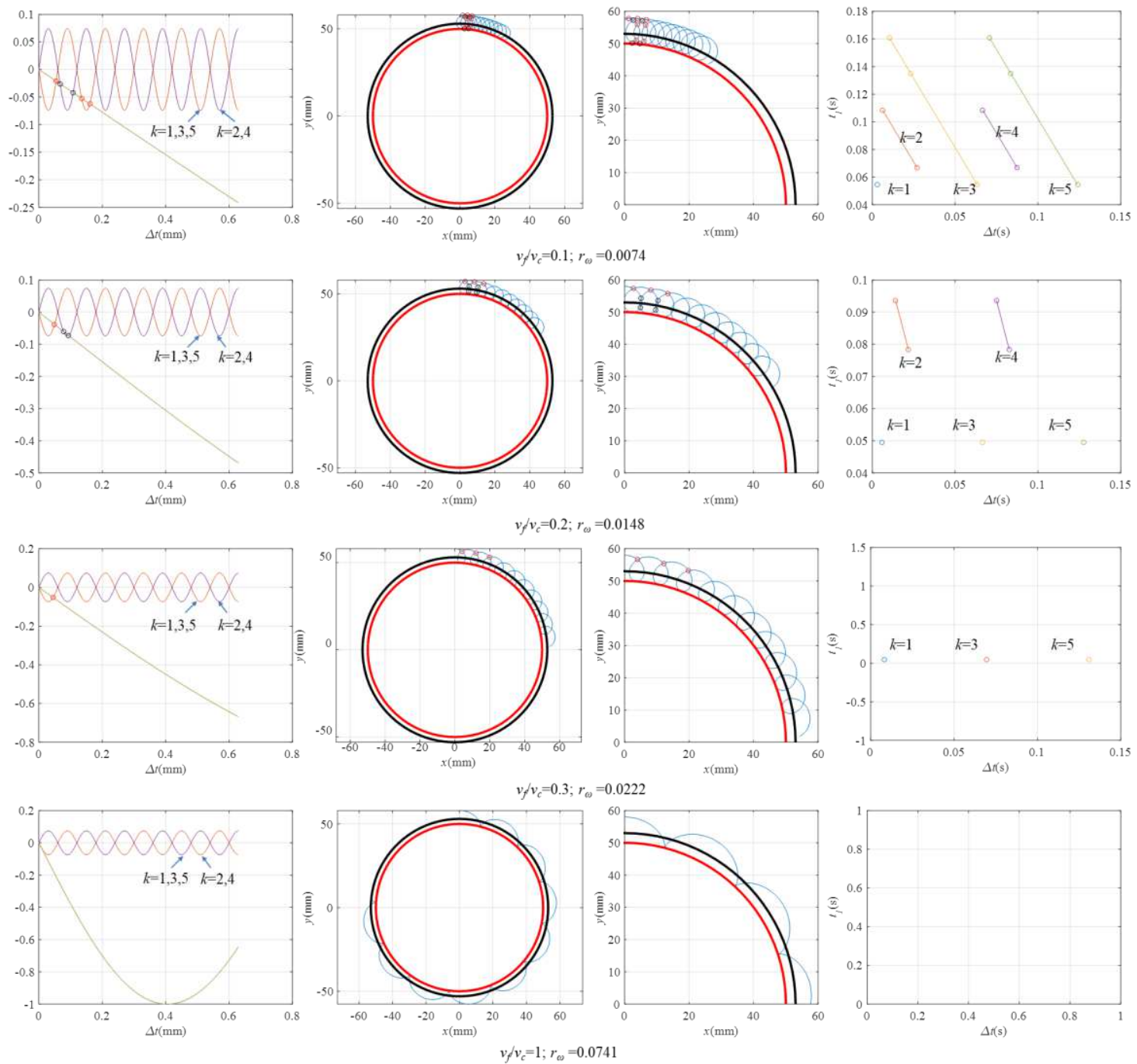

Fig. 26 Simulation of the self-intersection condition under different speed ratio ( $R=50 \mathrm{~mm}, r=4 \mathrm{~mm}, n=1000 \mathrm{rpm})$.

Under the condition of $r_{v}<r_{v c r i t i c a l}$, the desirable point that related to the roughness profile is found to be the first intersection point between the two sinusoidal curves (namely, $-\sin \left(\omega^{\prime} \Delta t / 2\right)$ and $(-1)^{k} r /(R+r) \sin (\omega \Delta t / 2), k$ is even $\left.)\right)$ as shown in the Fig. 27. This intersection can be easily computed numerically. The corresponding $t_{l}$ can also be obtained according to Eq. (28). The 
combination of $\Delta t$ and $t_{l}\left(\Delta t, t_{l}\right)$ that related to the roughness profile is shown in the last column of Fig. 26. As long as the desirable $t_{l}$ is obtained, the intersection point of the trajectory can be obtained and thus the arcs between two adjacent self-intersection points. The roughness profile can be finally constructed. Fig. 28 shows the machined roughness profile when $r=4 \mathrm{~mm}, n=1000$ $\mathrm{rpm}, v_{f} / v_{c}=0.05$.

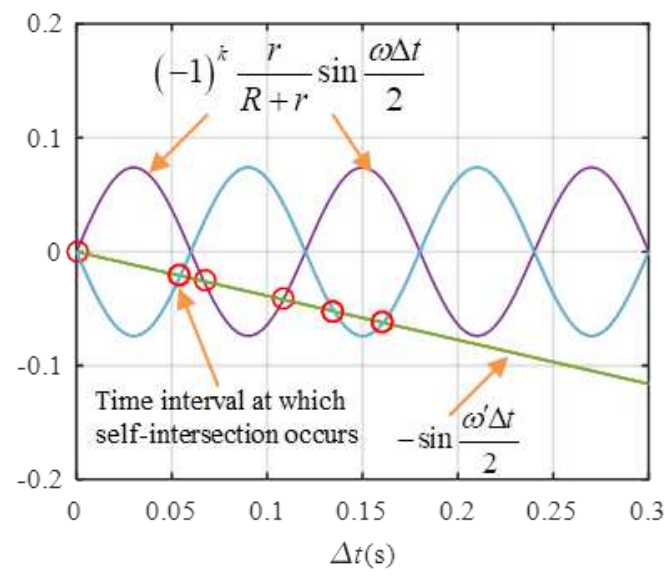

Fig. 27 Lowest self-intersection instant $\left(R=50 \mathrm{~mm}, r=4 \mathrm{~mm}, n=1000 \mathrm{rpm}, v_{f} / v_{c}=0.1\right)$.

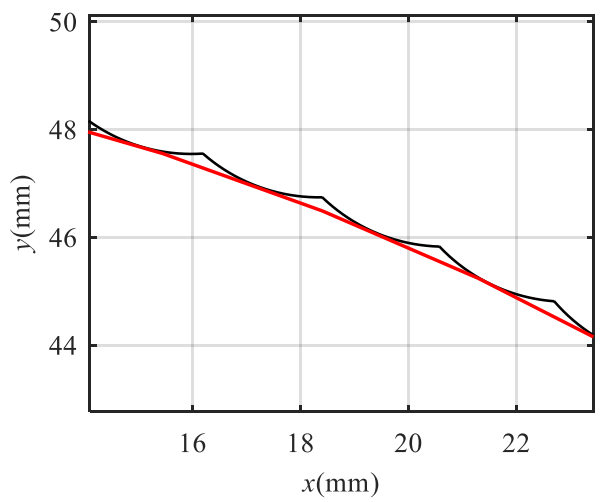

Fig. 28 The roughness profile when $R=50 \mathrm{~mm}, r=4 \mathrm{~mm}, n=1000 \mathrm{rpm}, v_{f} / v_{c}=0.05$.

The influence of the ratio between feed speed and cutting speed $r_{v}$ on the roughness profile when milling along a round path can be analyzed according to the above descripted method. The roughness profile of when $r_{v}$ has different values is shown in Fig. 29. From the figure, it can be seen that the profile becomes rough when the $r_{v}$ increases. It is similar to the case when milling along a straight path. 


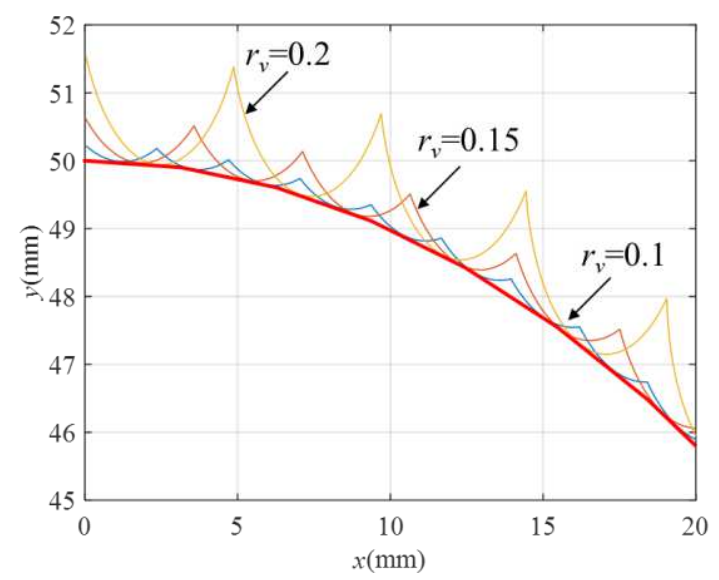

Fig. 29 The influence of ratio between the feed speed and cutting speed on roughness profile.

In Eq.(29), $r /(r+R)$ can be rewritten as $1 /(1+1 / r / R)$, which means that the roughness profile is depend not only upon the speed ratio of $r_{v}$ but also the ratio between the cutter radius and workpiece radius, namely $r_{r}=r / R$. The effect of radius on the roughness profile can also be numerically investigated according to the previous described method. Fig. 30 shows the roughness profile when $r_{r}$ has different values. From the figure, it can be found that the profile becomes rough when the $r_{v}$ increases, which means to obtain smoother surface when milling round workpiece cutter has smaller radius should be recommended.

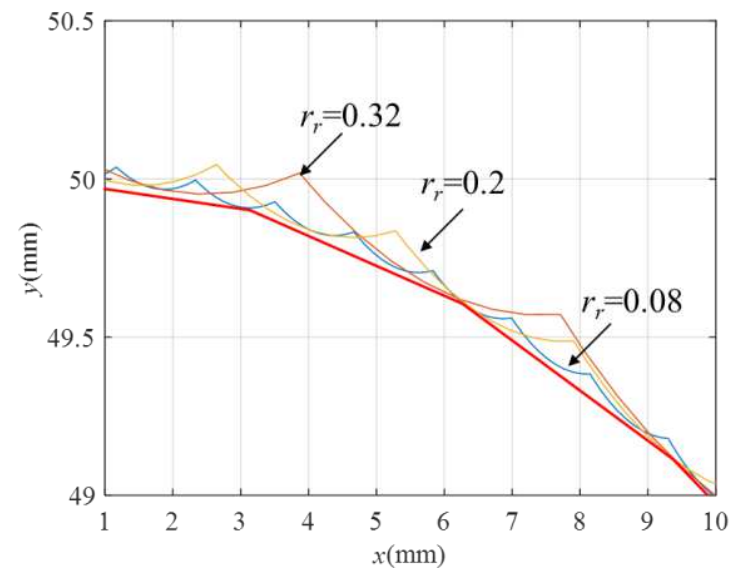

Fig. 30 The influence of ratio between the cutter radius and workpiece on roughness profile.

\subsection{Milling along a general curve path}

Workpieces with curved surface are also widely used in the engineering practice. The cutter will move along a curve path when milling the workpiece with curved surface (As shown in Fig. 31 (a)). In this part, the roughness profile of the workpiece with curved surface after milling is constructed. For simplicity, the cutter is assumed to be with a single and straight tooth. The moving path of the cutter center is assumed to be $(x(u), y(u))$. Two coordinate systems ( $x-O-y$ and 
$\left.x_{1}-O_{1}-y_{1}\right)$ as shown in the Fig. 31 (b) are constructed.

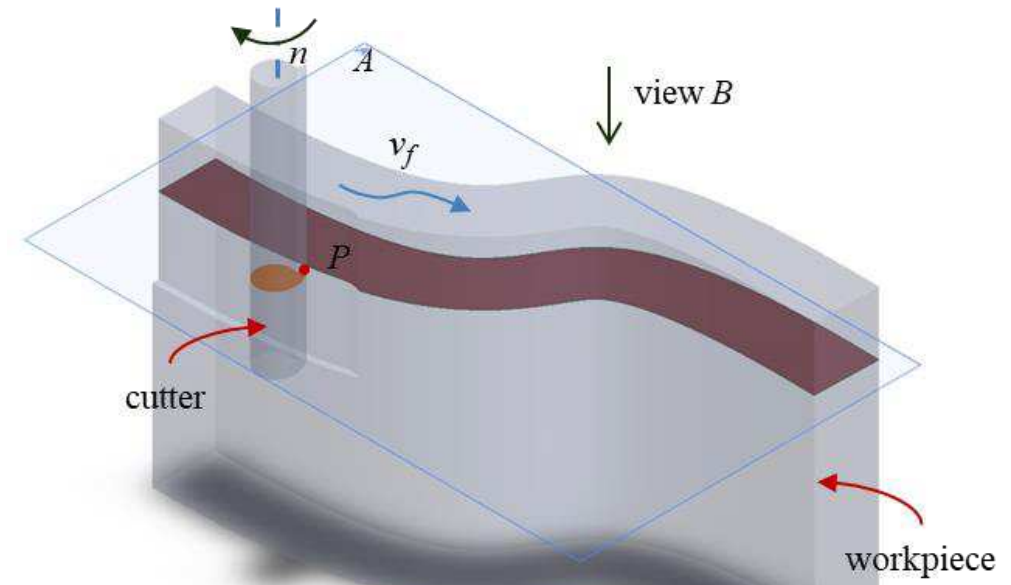

(a)

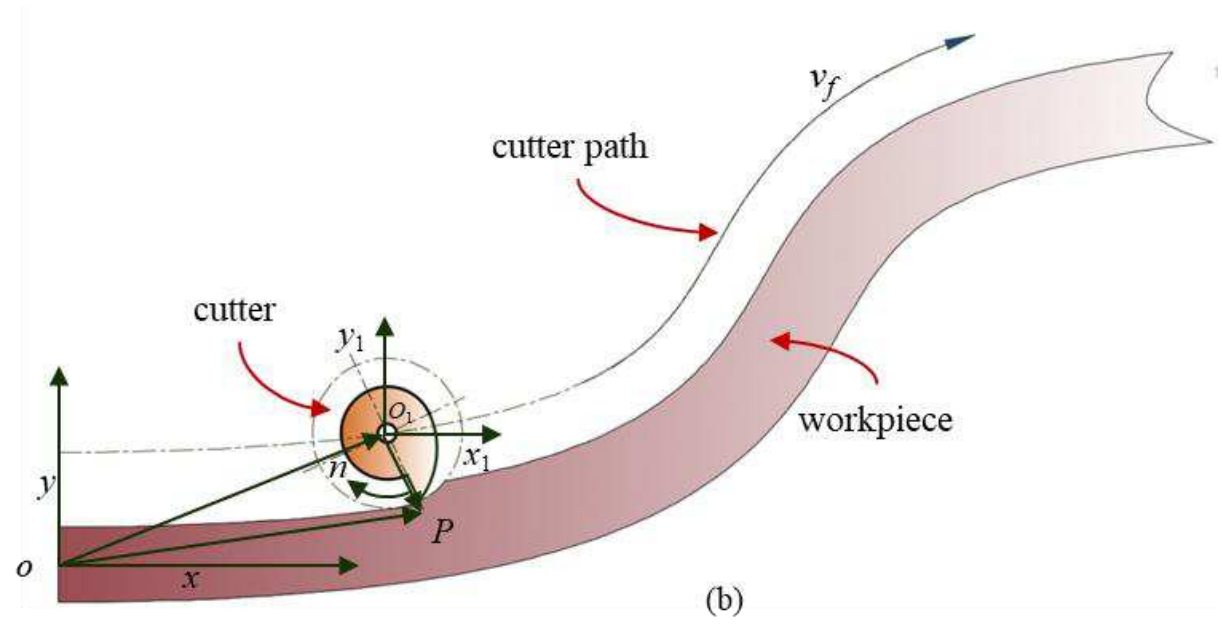

Fig. 31 Milling workpiece with complex surface: (a) 3D view during milling workpiece with curve surface; (b) section in the Plane A (View from B).

\subsubsection{The trajectory of a point on the cutting edge}

According to Fig. 31 (b), the trajectory of a point $P$ on the tooth edge is controlled by the following vector relationship

$$
\overline{O P}=\overline{O O^{\prime}}+\overline{O^{\prime} P}
$$

in which

$$
\left\{\begin{array}{l}
O O^{\prime}=(x(u), y(u)) \\
\overline{O^{\prime} P}=\left(r \sin \left(\theta+\theta_{0}\right), r \cos \left(\theta+\theta_{0}\right)\right)
\end{array}\right.
$$

Take the expressions of $\overline{O O_{1}}$ and $\overline{O_{1} P}$ into Eq.(31) results in

$$
\left\{\begin{array}{l}
x_{p}=x(u)+r \sin \left(\omega t+\theta_{0}\right) \\
y_{p}=y(u)+r \cos \left(\omega t+\theta_{0}\right)
\end{array}\right.
$$


If the feed speed is constant, the location of the cutter center $(x(u), y(u))$ after a time period $t$ is controlled by the following expression

$$
\int_{0}^{u} \sqrt{\left(x^{\prime}(u)\right)^{2}+\left(y^{\prime}(u)\right)^{2}} d u=v_{f} t
$$

the above equation can be rewritten as

$$
\frac{\sqrt{\left(x^{\prime}(u)\right)^{2}+\left(y^{\prime}(u)\right)^{2}}}{v_{f}}=\frac{d t}{d u}
$$

from which one can solve the relation between $t$ and $u$. Usually, it is impossible to get the analytical solution of $t$ respect to $u$, which means it is also impossible to get analytical expressions of $x(u)$ and $y(u)$

\subsubsection{Solve the self-intersection points and construct roughness profile}

To construct the roughness, the self-intersection points of the trajectory needs to be solved. Then the self-intersection points that related to the roughness profile needs to be extracted. Assuming that the coordinate of a self-intersection point is $\left(x_{1}, y_{1}\right)$, the time instant that the intersection occurs is $t_{1}$. After a time period of $\Delta t$, the point will go back to itself, which means that

$$
\left\{\begin{array}{l}
x\left(u\left(t_{1}\right)\right)+r \sin \left(\omega t_{1}+\theta_{o}\right)=x\left(u\left(t_{1}+\Delta t\right)\right)+r \sin \left(\omega\left(t_{1}+\Delta t\right)+\theta_{0}\right) \\
y\left(u\left(t_{1}\right)\right)+r \cos \left(\omega t_{1}+\theta_{o}\right)=y\left(u\left(t_{1}+\Delta t\right)\right)+r \cos \left(\omega\left(t_{1}+\Delta t\right)+\theta_{0}\right)
\end{array}\right.
$$

from which the following equation can be obtained

$$
-\tan \left(\omega t_{1}+\theta_{0}+\frac{\omega \Delta t}{2}\right)=\frac{y\left(u\left(t_{1}+\Delta t\right)\right)-y\left(u\left(t_{1}\right)\right)}{x\left(u\left(t_{1}+\Delta t\right)\right)-x\left(u\left(t_{1}\right)\right)}=\frac{\Delta y_{1}}{\Delta x_{1}}
$$

The relationship between the $t_{1}$ and $\Delta t$ can be obtained from Eq.(36). It is really difficult to give an analytical expression between $t_{1}$ and $\Delta t$ because of the existence of a transcendental function and the unknown analytical expressions of $x(u)$ and $y(u)$. All one can do is using numerical method.

Instead of trying to solve Eq.(36) analytically or numerically, this paper proposes an approximation method to obtain the self-intersection points that related to the roughness profile. The method is based on the simple case that discussed in the Section 2.

The curve path is divided into many small parts. Each small curve path has two end points. A line segment can be determined from these two end points. Thus, the curve milling path is now approximated by a bunch of line segments. As such, the self-intersection points of the trajectory 
obtained when milling along a curve path can be approximated by the ones when milling an approximated cutting path, which is composed by a bunch of small line segments. The self-intersection points of the trajectory when milling along a small line segment can be easily obtained by referring the simple case discussed in Section 2. Once the self-intersection points are obtained, the roughness profile can be constructed. The key for this method is to determine the length of the small line segment. It should large enough to let the trajectory when the cutter moves along the line segment to have self-intersection points, but it should be kept to be small to guarantee the approximation accuracy of the curve path. This paper recommends that the line segment has a length that the cutter moves in two periods of the cutter rotation.

According to the above descripted method, a specific case is studied, i.e. the roughness profile of a workpiece with curve surface after milling is simulated. The path to mill the workpiece is assumed to be [16]

$$
\left\{\begin{array}{l}
x(u)=20+190 u(1-u)^{2}+40 u^{2}(1-u)+80 u^{3} \\
y(u)=5+80 u^{2}(1-u)+25 u^{3}
\end{array} u \in[0,1]\right.
$$

The relationship between $t$ and the control parameter $u$ is obtained according to Eq.(34) and (37), which is written as

$$
\frac{\sqrt{\left(190(1-u)^{2}-300 u(1-u)+200 u^{2}\right)^{2}+\left(160 u(1-u)-5 u^{2}\right)^{2}}}{v_{f}}=\frac{d t}{d u}, u \in[0,1]
$$

from which the relationship between $t$ and $u$ is solved by the Runge-Kutta method [17]. Fig. 32 shows the relationship between $u$ and $t$ when the $v_{f}=20.94 \mathrm{~mm} / \mathrm{min}$. Thus, the cutter center location $x(u)$ and $y(u)$ can be obtained accordingly. The trajectory of the edge point $P$ can also obtained according to Eq.(32), as shown in Fig. 33. 


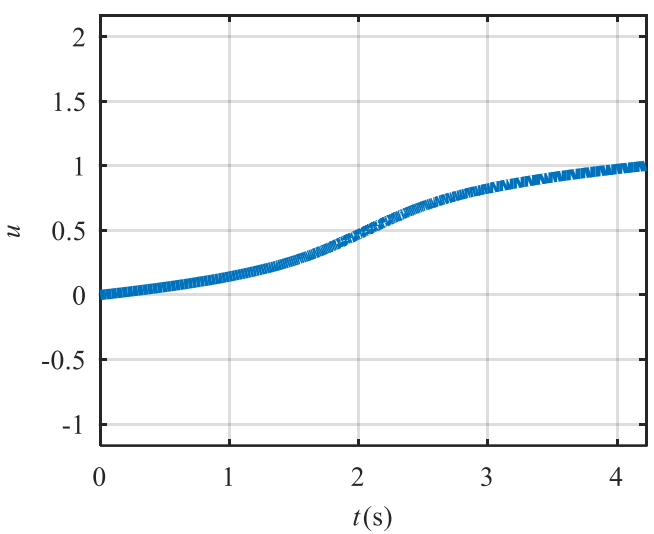

Fig. 32 The relationship between the $u$ and $t$.

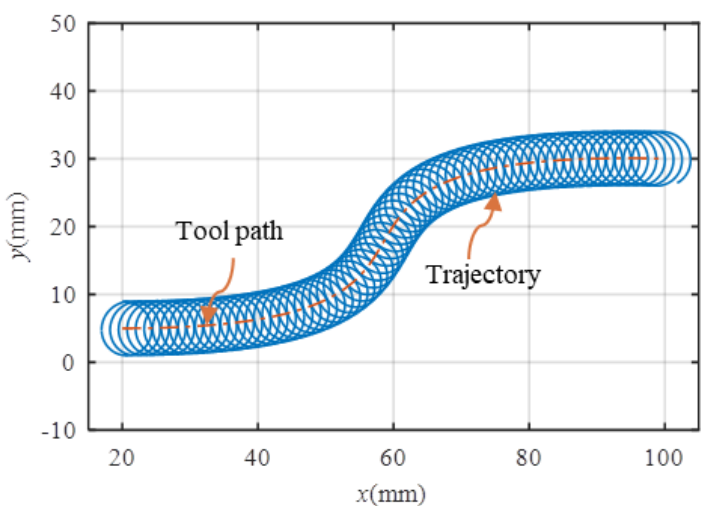

Fig. 33 The trajectory of the tooth edge point $\left(r=4 \mathrm{~mm}, n=1000 \mathrm{rpm}, v_{f} / v_{c}=0.05\right)$.

To construct the roughness profile, the self-intersection points that are related to the roughness profile are solved according to the proposed method. The results are shown in Fig. 34. The trochoidal arcs between two adjacent self-intersection points as shown in Fig. 34 can also be obtained according to the trajectory Eq.(32), the results are shown in Fig. 35 (a) and (b). The roughness profile is finally constructed as shown in Fig. 35 (c) and (d).
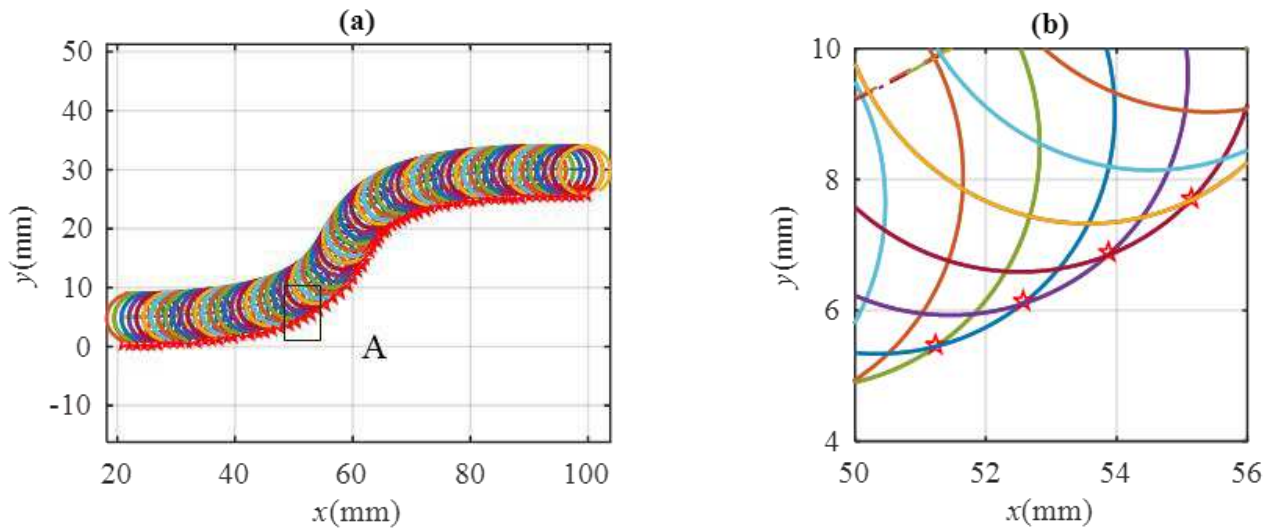

Fig. 34 The self-intersection points that are related to the roughness profile ( $\left.r=4 \mathrm{~mm}, n=1000 \mathrm{rpm}, v_{f} / v_{c}=0.05\right)$ : (a) all self-intersection points; (b) magnification of A. 
(a)

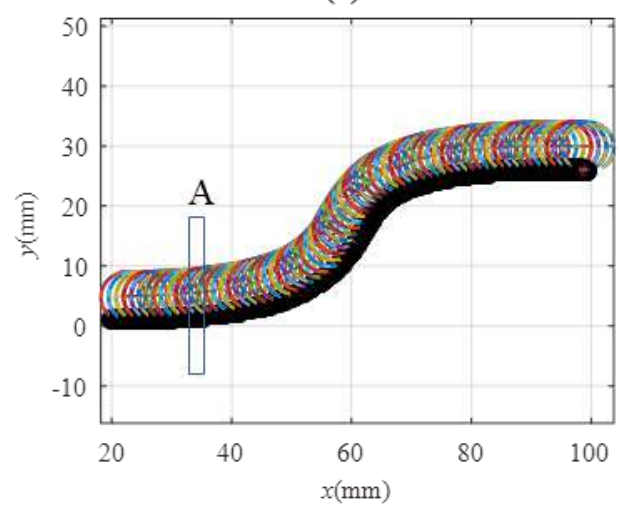

(c)

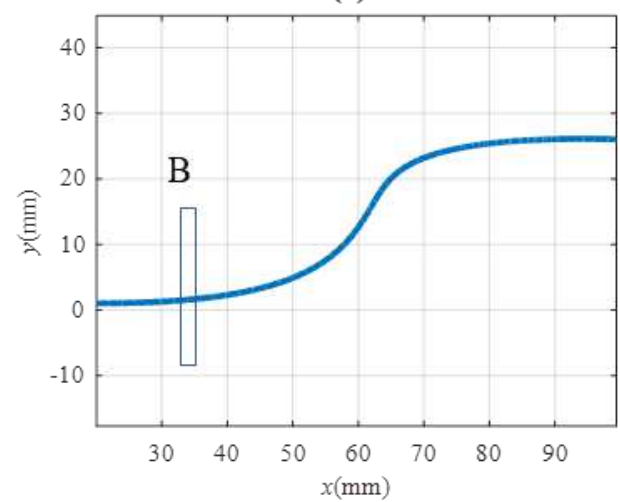

(b)

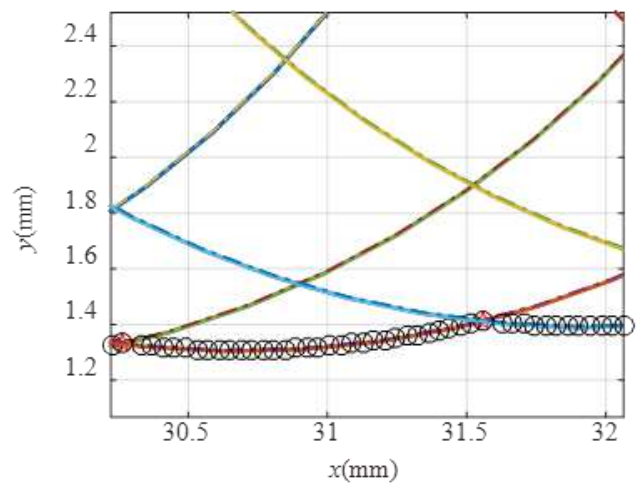

(d)

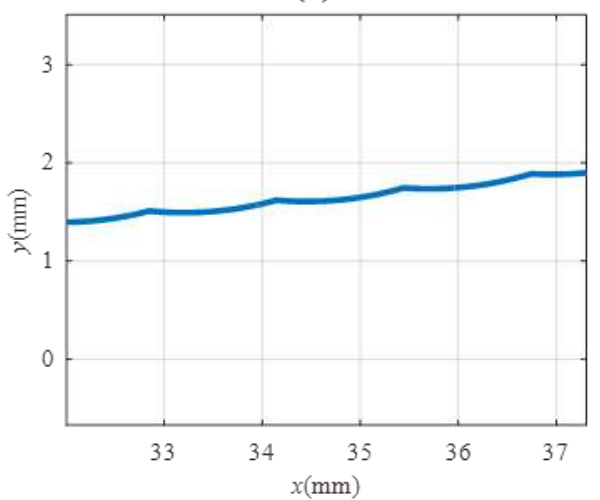

Fig. 35 The roughness profile on the curve path: (a) roughness profile with trajectory; (b) magnification of A; (c) roughness profile without trajectory; (d) magnification of B ( $r=4 \mathrm{~mm}, n=1000 \mathrm{rpm}, v_{f} / v_{c}=0.05$ ).

\section{Verification and discussion}

\subsection{Experimental demonstration}

The verification of the modeled roughness profile is conducted according to the following procedures: (i) several cutting experiments with different cutting parameters (as shown in Table 1) are carried out; (ii) the roughness profiles of the machined surface are tested; (iii) roughness profile under same cutting conditions used in the cutting experiment is simulated according to the procedures presented in the previous sections; (iv) compare the tested roughness profiles with the simulated ones.

A new end mill with radius equals to $3 \mathrm{~mm}$ is used in cutting experiments. The tool has four teeth and a helical of $45^{\circ}$. The material for the cutter is carbide. The workpiece used in the experiment is Aluminum blocks. After cutting, the roughness profile is tested under a telescope (Nano View). Meanwhile, the profile is simulated according to the procedures presented in the Section 4.1. And the simulated roughness profile and the tested roughness profile are compared. 
The comparison results are shown in Fig. 36. For all these tests, the predicted profile matches well with the tested one, which validates the rightness of the profile modeling method presented in this paper.
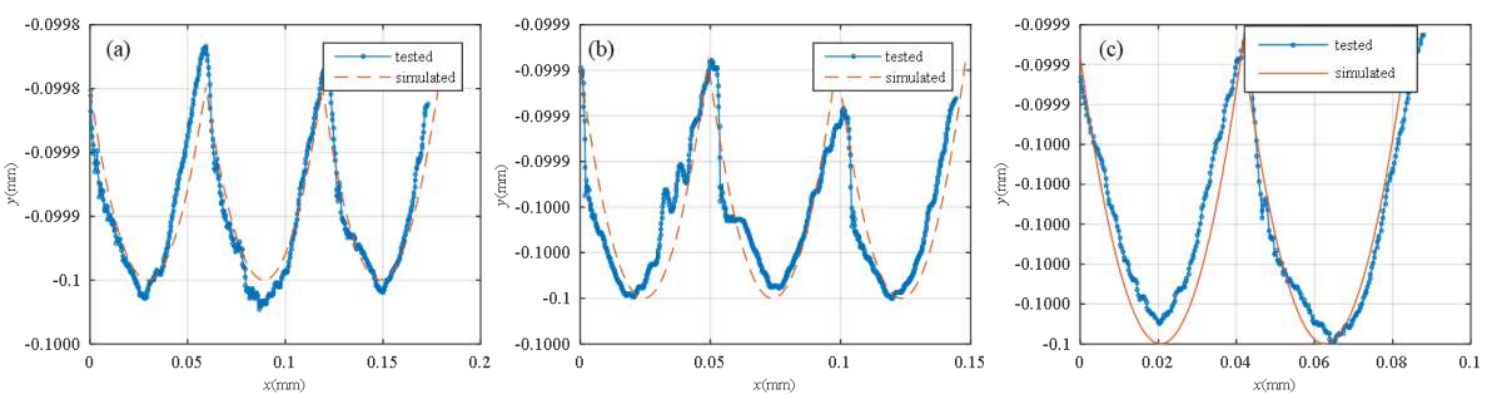

Fig. 36 Comparison between the simulated roughness profile and the tested one:(a) $n=2500 \mathrm{rpm}, a_{p}=3 \mathrm{~mm} ; a_{e}=0.1$ $\mathrm{mm}, v_{f}=600 \mathrm{~mm} / \mathrm{min}$; (b) $n=3000 \mathrm{rpm}, a_{p}=3 \mathrm{~mm} ; a_{e}=0.1 \mathrm{~mm}, v_{f}=600 \mathrm{~mm} / \mathrm{min}$; (c) $n=3000 \mathrm{rpm}, a_{p}=3 \mathrm{~mm} ; a_{e}=0.1$ $\mathrm{mm}, v_{f}=600 \mathrm{~mm} / \mathrm{min}$.

The cutting conditions are listed on the following table.

Table 2 Cutting conditions used during milling process.

\begin{tabular}{ccccc}
\hline $\begin{array}{c}\text { Group } \\
\text { number }\end{array}$ & $\begin{array}{c}\text { Spindle speed } n \\
(\mathrm{r} / \mathrm{min})\end{array}$ & $\begin{array}{c}\text { Axial depth of cut } \\
a_{p}(\mathrm{~mm})\end{array}$ & $\begin{array}{c}\text { Radial depth of cut } \\
a_{e}(\mathrm{~mm})\end{array}$ & $\begin{array}{c}\text { Feed velocity } v_{f} \\
(\mathrm{~mm} / \mathrm{min})\end{array}$ \\
\hline 1 & 2500 & 3 & 0.1 & 600 \\
2 & 3000 & 3 & 0.1 & 600 \\
3 & 3500 & 3 & 0.1 & 600 \\
\hline
\end{tabular}

\subsection{Compare the roughness profile construction method developed in this paper to the}

\section{existed one}

The construction method of the roughness profile developed in this method is compared to the one proposed by Arizemendi et al. [2,1]. The cutting tool used during simulation is a milling cutter with three flutes and radius of $4 \mathrm{~mm}$. The cutting parameters used in simulation are: spindle speed $n=1000 \mathrm{rpm}$, feed speed $v_{f}=300 \mathrm{~mm} / \mathrm{min}$. The MATLAB software is used to implement the simulation. The computer used to run the MATLAB simulation program has a processor of i7-3470 CPU@2.7 GHz and a RAM of 16 GB.

For the method developed in this paper, the time to construct a roughness profile that has a length of $3 \mathrm{~mm}$ is $0.011082 \mathrm{~s}$. For the method developed by Arizemendi et al. [2,1], the time needs to construct a roughness profile that has the same length is dependent on the distance between two 
adjacent vertical lines that cross the trajectories. Three different distance values, namely $0.1 \mathrm{~mm}$, $0.05 \mathrm{~mm}$ and $0.01 \mathrm{~mm}$, are chosen to execute the simulation. The time needs to construct the roughness profile for three different cases are $469.173782 \mathrm{~s}, 741.994641 \mathrm{~s}, 3567.835404 \mathrm{~s}$ respectively, which is much longer than the method developed in this paper. The roughness profiles obtained under these three different distances are listed Fig. 37 and compared to the one obtained by the method developed in this paper. It can be seen that the accuracy of the roughness profile obtained for the method developed by Arizemendi et al. [2,1] is related to the vertical distance. To obtaine accurate roughness profile, small distance is needed and thus more time will be consumed to construct the profile.

(a)

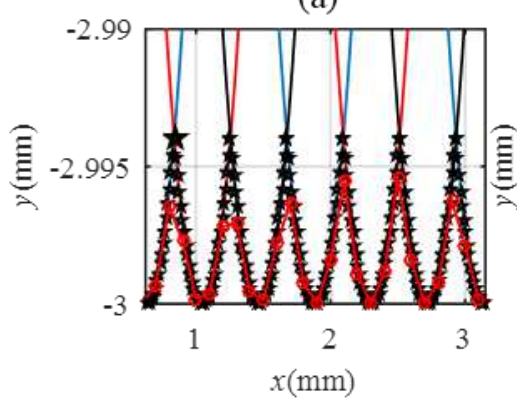

(b)

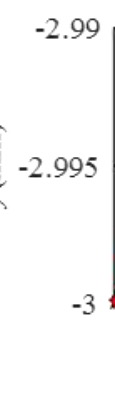

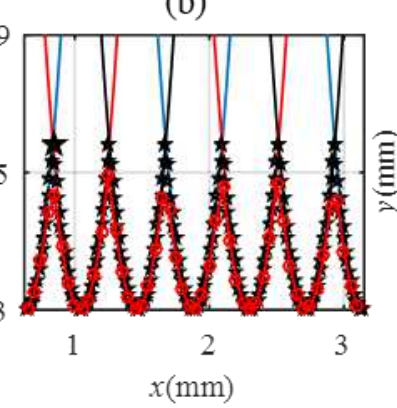

(c)

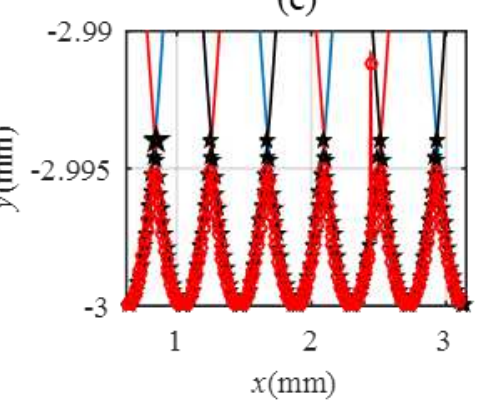

Fig. 37 Comparison between the roughness profile by the method developed in this paper and the one by Arizemendi et al. $[2,1](-$ means the trajectories, $-\star-$ means roughness profile the developed in this paper, o- means the roughness profile obtained by Arizemendi et al. [2,1]):(a) vertical distance equals to $0.1 \mathrm{~mm}$; (b) vertical distance equals to $0.05 \mathrm{~mm}$; (c) vertical distance equals to $0.01 \mathrm{~mm}$.

\section{Conclusions}

In this paper, the roughness profile generated by flank milling process is theoretically investigated. For the first time, the roughness profile is modeled and analyzed analytically. This is the main difference between this investigation and the existed ones. Based on the constructed the roughness profile model, the effect of the cutting parameters, the cutter geometry parameters on the roughness profile is discussed. Besides, the roughness profile of the machined surface obtained when the cutting tool feeds along curve paths are also constructed. At last, experiments are conducted to verify the developed method. The main conclusions can be summarized as following:

- Two parameters, namely $w$ and $h$ can be defined to descript the roughness profile 
obtained when the cutter moves along a straight path, in which $w$ means the width of the elemental profile and $h$ means the height of the roughness profile.

- The height of the roughness profile $h$ is related to the ratio between feed speed and the cutting speed. $h$ will increase with the speed ratio. This is true when $h$ is smaller than the radial depth of cut. When the $h$ is larger than the radial depth of cut, the shape of roughness profile will be decided by the ratio as well as the radial depth of cut.

Both $w$ and $h$ will decrease as the increasing of tooth number for a milling cutter with equal tooth spacing angle.

- The roughness profile of the milling cutter with variable pitch is also simulated. The height of the roughness profile $h$ becomes larger compared to the cutter with equal pitch.

- The helical angle has no effect on the profile parameters $w$ and $h$. But it will affect the surface topography. The peaks and valley of the surface topography lie on a bunch of slope lines.

- The roughness profiles when the cutter feeds along a round path and a curve path are also simulated. It is impossible to analytically solve the self-intersection points that are used to construct the roughness profile. Thus, a numerical method is proposed. And the roughness profile is successfully constructed.

- The roughness profile measured from the machined surface matches well with the simulated one, which demonstrates the correctness of the roughness profile construction method developed in this paper.

- The method to construct the surface roughness developed in this paper is compared to the one developed by Arizemendi et al. The method in this paper consumes much less time to construct the surface roughness.

In the future investigation, more attention will be focused on the effect of other factors such as the tool vibration, tool wear, runout etc. on the roughness profile and surface topography based on the method proposed in this paper.

\section{Declarations}

Funding The work is supported by the internal projects No.****-1922-SX and the National Basic Research Program (973 Program, Grant No. 2014CB046603). Besides, the theoretically part of 
this paper was finished in the Manufacturing \& Automation Research Laboratory, Rutgers

University, USA. during which the author work as a visiting student supported by the National Scholarship Council of China P.R.

Conflicts of interest The authors declare no competing interests.

Availability of data and materials The data and materials of this manuscript are available from the corresponding author on reasonable request.

Code availability Not applicable

Ethical Approval Not applicable

Consent to Participate Not applicable

Consent to Publish All authors are consent to publish this manuscript in The International Journal of Advanced Manufacturing Technology.

\section{References}

1. Arizmendi M, Campa FJ, Fernández J, López de Lacalle LN, Gil A, Bilbao E, Veiga F, Lamikiz A (2009) Model for surface topography prediction in peripheral milling considering tool vibration. CIRP Ann-Manuf 58 (1):93-96. doi:http://dx.doi.org/10.1016/j.cirp.2009.03.084

2. Arizmendi M, Fernández J, Lacalle LNLd, Lamikiz A, Gil A, Sánchez JA, Campa FJ, Veiga F (2008) Model development for the prediction of surface topography generated by ball-end mills taking into account the tool parallel axis offset. Experimental validation. CIRP Ann-Manuf 57 (1):101-104. doi:http://dx.doi.org/10.1016/j.cirp.2008.03.045

3. Quinsat Y, Sabourin L, Lartigue C (2008) Surface topography in ball end milling process: Description of a 3D surface roughness parameter. J Mater Process Technol 195 (1-3):135-143. doi:http://dx.doi.org/10.1016/j.jmatprotec.2007.04.129

4. Layegh K SE, Lazoglu I (2017) 3D surface topography analysis in 5-axis ball-end milling. CIRP Ann-Manuf 66 (1):133-136. doi:https://doi.org/10.1016/j.cirp.2017.04.021

5. Arizmendi M, Jiménez A (2019) Modelling and analysis of surface topography generated in face milling operations. Int J Mech Sci 163:105061. doi:https://doi.org/10.1016/j.ijmecsci.2019.105061

6. Buj-Corral I, Vivancos-Calvet J, González-Rojas H (2011) Influence of feed, eccentricity and helix angle on topography obtained in side milling processes. Int J Mach Tools Manuf 51 (12):889-897. 
doi:https://doi.org/10.1016/j.ijmachtools.2011.08.001

7. Chang C (1992) Mathematical modeling and analysis of the surface topography generated during end milling processes. Master Thesis, The University of Maryland, Maryland

8. Zhang C, Guo S, Zhang H, Zhou L (2013) Modeling and predicting for surface topography considering tool wear in milling process. Int $J$ Adv Manuf Technol 68 (9):2849-2860. doi:10.1007/s00170-013-4989-7

9. Omar OEEK, El-Wardany T, Ng E, Elbestawi MA (2007) An improved cutting force and surface topography prediction model in end milling. Int $\mathrm{J}$ Mach Tools Manuf 47 (7):1263-1275. doi:https://doi.org/10.1016/j.ijmachtools.2006.08.021

10. Arizmendi M, Fernández J, Gil A, Veiga F (2009) Effect of tool setting error on the topography of surfaces machined by peripheral milling. Int J Mach Tools Manuf 49 (1):36-52. doi:https://doi.org/10.1016/j.ijmachtools.2008.08.004

11. Surmann T, Biermann D (2008) The effect of tool vibrations on the flank surface created by peripheral milling. CIRP Ann-Manuf 57 (1):375-378. doi:http://dx.doi.org/10.1016/j.cirp.2008.03.059 12. Stepan G, Hajdu D, Iglesias A, Takacs D, Dombovari Z (2018) Ultimate capability of variable pitch milling cutters. CIRP Ann-Manuf 67 (1):373-376. doi:https://doi.org/10.1016/j.cirp.2018.03.005 13. Altıntaş Y, Engin S, Budak E (1999) Analytical Stability Prediction and Design of Variable Pitch Cutters. J Manuf Sci Eng 121 (2):173-178. doi:10.1115/1.2831201

14. Sims ND, Mann B, Huyanan S (2008) Analytical prediction of chatter stability for variable pitch $\begin{array}{llllll}\text { and variable helix milling tools. J Sound Vib } 317 & \text { (3):664-686. }\end{array}$ doi:https://doi.org/10.1016/j.jsv.2008.03.045

15. Shirase K, Altintaş Y (1996) Cutting force and dimensional surface error generation in peripheral milling with variable pitch helical end mills. Int J Mach Tools Manuf 36 (5):567-584. doi:https://doi.org/10.1016/0890-6955(95)00063-1

16. Yang Y, Zhang WH, Ma YC, Wan M (2016) Chatter prediction for the peripheral milling of thin-walled workpieces with curved surfaces. Int J Mach Tools Manuf 109:36-48. doi:http://dx.doi.org/10.1016/j.ijmachtools.2016.07.002

17. Dormand JR, Prince PJ (1980) A family of embedded Runge-Kutta formulae. J Comput Appl Math 6 (1):19-26. doi:https://doi.org/10.1016/0771-050X(80)90013-3 


\section{Appendix}

Derivation of Eq.(7)

Square on both sides of each equation for Eq. (4) results in

$$
\left\{\begin{array}{l}
r^{2} \sin ^{2} \varphi=\left[v_{f} \Delta t+r \sin (\varphi+\Delta \varphi)\right]^{2} \\
r^{2} \cos ^{2} \varphi=r^{2} \cos ^{2}(\varphi+\Delta \varphi)
\end{array}\right.
$$

Add each equation on both sides of Eq. (A.1) results in

$$
v_{f} \Delta t+r \sin (\varphi+\Delta \varphi)=0
$$

which can be written as

$$
\sin (\varphi+\Delta \varphi)=-\frac{v_{f} \Delta t}{r}
$$

Because that $\omega \Delta t=\Delta \varphi$, substituting $\Delta t=\Delta \varphi / \omega$ into Eq. (A.3) results in

$$
\sin (\varphi+\Delta \varphi)=-\frac{v_{f} \Delta \varphi}{\omega r}
$$

Let $v_{c}=\omega r$, Eq. (A.4) becomes

$$
\sin (\varphi+\Delta \varphi)=-\frac{v_{f} \Delta \varphi}{v_{c}}
$$

